\title{
Ribosomal ubiquitination facilitates mRNA cleavage and ribosome rescue during No-Go and Nonstop mRNA Decay
}

4 Parissa C. Monem ${ }^{1}$, Audrey L. Piatt ${ }^{1}$, Nitin Vidyasagar ${ }^{1}$, Marissa L. Glover ${ }^{1}$, Thea A. Egelhofer ${ }^{1}$, 5 Joshua A. Arribere, 1, ,

7 'Department of Molecular, Cell, and Developmental Biology, University of California at Santa

8 Cruz, Santa Cruz, CA, USA

'Lead Contact

*Correspondence: jarriber@ucsc.edu

\section{ABSTRACT}

During translational surveillance, ribosomes play a critical role in detecting problematic mRNAs and signaling cellular machinery to repress the offending messages. Prior work has shown that problematic mRNAs identified by two surveillance pathways (Nonstop and No-Go mRNA Decay) are detected by ribosome collisions and subsequent ribosomal ubiquitination, yet how ribosomal ubiquitination leads to repression has remained unclear. Here, we deploy $C$. elegans to unravel the series of coordinated events during Nonstop and No-Go mRNA Decay. We probe 9 the metazoan SKI RNA helicase complex to uncover functionally significant residues and reveal divergence of the SKI-exosome interface. We define a functional requirement for ubiquitination on at least two ribosomal proteins during No-Go mRNA Decay, and illustrate how ubiquitination recruits the endonuclease NONU-1 via CUE domains and the ribosome rescue factor HBS-1 via its poorly characterized $\mathrm{N}$-terminus. Our molecular characterization (1) underscores the importance of ribosomal ubiquitination in mRNA degradation, (2) shows similar and distinct genetic dependencies of factors in Nonstop and No-Go mRNA Decay, and (3) uncovers a conspicuous absence of distinct ribosomal stalls at No-Go mRNA Decay substrates. Our work demonstrates mechanisms by which translation signals to effectors of co-translational mRNA repression and has implications for the study of translation and ribosomal species in vivo. 


\section{INTRODUCTION}

An organism's growth, function, and response to environmental changes rely on ribosomes accurately producing proteins. Subtle errors in mRNAs are often elusive, requiring active translation to detect the error and trigger downstream repression. If left unchecked, defective mRNAs have the potential to produce toxic proteins resulting in disease phenotypes, such as neurodegeneration seen in mice (Ishimura et al., 2014; Bengtson and Joazeiro, 2010). Ribosomes that translate problematic mRNAs can elicit various repressive mechanisms, thus ensuring proper gene expression. Two such mechanisms are Nonstop mRNA Decay and NoGo mRNA Decay. Nonstop mRNA Decay is triggered by mRNAs lacking stop codons, which are formed by premature polyadenylation or mRNA cleavage (Frischmeyer et al., 2002). No-Go mRNA Decay occurs on mRNAs containing a variety of elongation-inhibiting features, such as stable secondary structures, stretches of rare codons, polybasic amino acid-encoding sequences, or damaged nucleotides (Doma and Parker, 2006). While triggered by different mRNA species, both of these pathways result in mRNA decay, ribosome rescue, and nascent peptide degradation through the coordinated recruitment of several effectors.

Two modes of mRNA repression are exonucleolytic decay and endonucleolytic cleavage. Key to exonucleolytic decay is the SKI RNA helicase complex, which is required for mRNA decay in multiple translational surveillance pathways (Frischmeyer et al., 2002; Doma and Parker, 2006; Arribere and Fire, 2018). Two non-mutually exclusive models for SKI function exist: one points to a role in loading the 3'>5' exosome to degrade offending mRNAs (Araki et al., 2001; van Hoof et al., 2002; Doma and Parker, 2006; Hashimoto et al., 2017; Kowalinski et al., 2016), while another places the SKI complex acting alone by extracting RNA from ribosomes (Zinoviev et al., 2020). Which of these functions is essential to SKI-mediated repression remains unclear. Endonucleolytic cleavage can initiate mRNA repression through downstream decay by cellular exonucleases. Recently identified in both $C$. elegans and S. cerevisiae, NONU1/Cue2/YPL199C are endonucleases responsible for cleaving targets of Nonstop and No-Go mRNA Decay in the vicinity of stalled ribosomes (Glover et al., 2020; D'Orazio et al., 2019). While NONU-1 is required for efficient Nonstop and No-Go mRNA Decay, it remains unclear how and when the factor is targeted to stall-inducing mRNAs. However, clues to its recruitment mechanism exist: NONU-1 and its homologs contain at least two conserved ubiquitin-binding 60 domains. 
During both Nonstop and No-Go mRNA Decay, ribosomes on problematic mRNAs are rescued and their nascent peptides are degraded. HBS-1 is a GTPase homologous to eRF3 and is thought to function in ribosome rescue by loading PELO-1/Dom34 (homologous to eRF1) in a codon-independent manner (Doma and Parker, 2006; Shoemaker et al., 2010; Becker et al., 2011; Saito et al., 2013; Hilal et al., 2016). Recruitment of PELO-1 leads to ribosome subunitsplitting by the AAA ATPase ABCE-1 and the eventual proteasomal degradation of the nascent peptide (Pisareva et al., 2011; Shao et al., 2014). While the precise recruitment mechanism of HBS- 1 and PELO- 1 is assumed to be via PELO-1 recognition of an empty ribosomal A-site, additional data showing HBS-1/PELO-1 acting on internally-stalled ribosomes challenges this model (Pisareva et al., 2011).

Recent advances have been made towards characterizing the early steps of No-Go mRNA Decay. One key upstream event is the generation of a stalled, collided ribosome species at a problematic site on the mRNA. This species is thought to be unique to No-Go mRNA Decay and could conceivably recruit downstream effectors (Simms et al., 2017; Juszkiewicz et al., 2018; Ikeuchi et al., 2019). The interface between collided ribosomes is the site of ubiquitination events on multiple ribosomal proteins near the mRNA's path (Juszkiewicz et al., 2018; Ikeuchi et al., 2019). The conserved E3 ubiquitin ligase ZNF-598 is thought to deposit ubiquitin marks on at least two ribosomal proteins, including RPS-10 (eS10) and RPS-20 (uS10) (Juszkiewicz \& Hegde, 2017; Sundaramoorthy et al., 2017). These ubiquitin marks are thought to act as signaling mechanisms for further events.

Prior work highlights the importance of ribosomal ubiquitination in No-Go mRNA Decay, but has yet to fully understand its downstream consequences. This limitation is in part due to the difficulty in recovering viable mutants of the target ribosomal proteins, which has thus complicated a straightforward analysis of individual ubiquitination sites. Harnessing a system to study the functional contributions of ribosomal ubiquitination is likely to clarify downstream events. While structural studies revealed multiple collided ribosome structures with ubiquitination sites at the collision interface, the relationship of these collided structures to in vivo stalls on mRNAs remains poorly understood. Several recent studies have mapped the

92 locations of ribosome species thought to represent collisions, known as disomes or di-

93 ribosomes (Meydan et al., 2020; Han et al., 2020; Arpat et al., 2020; Zhao et al., 2021).

94 However, the application of these data to No-Go mRNA Decay is lacking, in part due to the 95 absence of work containing validated reporters and the lack of bonafide endogenous No-Go 
mRNA Decay targets. Additionally, our current understanding of ribosomal ubiquitination by ZNF-598 relies on work utilizing reporters with stalls akin to No-Go mRNA Decay exclusively (Garzia et al., 2017; Juszkiewicz and Hegde, 2017; Sundaramoorthy et al., 2017). Given that Nonstop and No-Go mRNA Decay arise on distinct mRNA substrates, a more complete illustration of ribosomal ubiquitination function requires a consideration of both pathways. Decay. Unlike most metazoans, mutants of surveillance and decay machinery are viable in $C$. elegans, making it a genetically tractable system for this work. Through our Nonstop mRNA Decay reporter, we probed the function of the metazoan SKI complex and provided support for exosome-independent mRNA repression. We uncovered a functional role for ribosomal ubiquitination by ZNF-598 in recruiting both NONU-1 and HBS-1 to stalled ribosomes during NoGo mRNA Decay, revealing a novel and conserved Ub-binding function for the poorly characterized N-terminus of HBS-1. Additionally, we found that site-specific mutation of ribosomal ubiquitination targets RPS-10 (eS10) and RPS-20 (uS10) together phenocopy knock out of ZNF-598, and are required to elicit full No-Go mRNA Decay. Interestingly, while we observed a requirement of HBS-1/PELO-1 and NONU-1 in both Nonstop and No-Go mRNA Decay, we uncovered differential dependencies on ZNF-598, suggesting an alternative E3 ligase acts in Nonstop mRNA Decay. Our subsequent results via RNA-seq and Ribo-seq substantiated the importance of ZNF-598 in destabilizing No-Go mRNAs and shed light on the molecular dynamics of ribosomal stalling.

\section{RESULTS}

\section{Novel Nonstop mRNA Decay suppressors identify functionally important residues in the} SKI complex

To characterize the genetic requirements of Nonstop mRNA Decay, we used a Nonstop mRNA Decay reporter we previously constructed by CRISPR/Cas9 (Fig 1A) (Arribere and Fire, 2018). Briefly, the reporter encodes the muscle myosin unc-54, GFP, a T2A 'stop-and-go' peptide, and no in-frame stop codons. Strains with the unc-54(nonstop) construct efficiently degrade mRNAs

125 from this locus via Nonstop mRNA Decay, conferring little movement and very low GFP

126 fluorescence, whereas strains with a compromised Nonstop mRNA Decay pathway exhibit

127 increased movement and GFP fluorescence. In prior work, we used the unc-54(nonstop)

128 reporter to identify seventeen mutations in the Nonstop mRNA Decay machinery. Here, we 
report the identification of an additional fourteen mutations, starting with twelve mutations in SKI complex subunits (Fig 1B).

Of the twelve mutations in SKI complex subunits, eight were in skih-2 (S. cer SKI2) and four were in $t t c-37$ (S. cer SKI3). The skih-2 mutations were exclusively missense mutations and hit residues conserved from $S$. cerevisiae to humans (Fig S1). The majority of the skih-2 suppressors were in the catalytic RecA domains of this protein, or lined the mRNA binding channel (Fig S1). Identification of these mutations is consistent with mRNA binding and catalysis by the SKIH-2 helicase as being required for Nonstop mRNA Decay, consistent with prior work across experimental systems. We expect that future work with these mutations will further our understanding of SKI function. Two mutations (G363E and G367E) were close to the interface of Ski2 and Ski8; existence of such mutations suggests that this interface and/or Ski8 may also be important in $C$. elegans, though we did not identify a single mutation in a factor that could conceivably function as Ski8 (see below). All four of the novel ttc-37 suppressors were premature stop codons and will not be discussed further.

Our subsequent analysis of the $t t c-37$ and skih-2 genes agreed with a model where these two genes function together as a complex. We observed a similar fold de-repression in ttc-37 and skih-2 mutants (Fig 1C). Furthermore, we observed epistasis: a skih-2 ttc-37 double mutant exhibited a similar fold de-repression to the single mutants (Fig 1C). These results are consistent with SKI complex function requiring the activity of both Ski2/Ski3-like subunits, in line with how this complex is thought to function in yeast and humans (Halbach et al., 2013; Kalisiak et al., 2017).

\section{A novel ribosome stalling screen identifies core No-Go mRNA Decay machinery}

154 To establish C. elegans as a system to study No-Go mRNA Decay, we built a ribosome stalling 155 reporter using CRISPR/Cas9 (Fig 1D). As with our Nonstop mRNA Decay reporter, we started 156 with the unc-54 locus and C-terminally added a T2A 'stop-and-go' peptide, FLAG, twelve rare 157 arginine codons, and GFP followed by a stop codon. We selected rare arginine codons for two 158 reasons: (1) arginine is a positively charged amino acid and prior work (Lu and Deutsch, 2008;

159 Chandrasekaran et al., 2019) suggests this may induce ribosome stalling via interactions with 160 the peptide exit tunnel, and (2) the Arg-tRNAs decoding two of the rarest codons (CGG, AGG) 161 in C. elegans are very lowly expressed under normal growth conditions (Aidan Manning, Todd 162 Lowe, personal communication, June 2021), providing a second avenue by which stalling may 
A
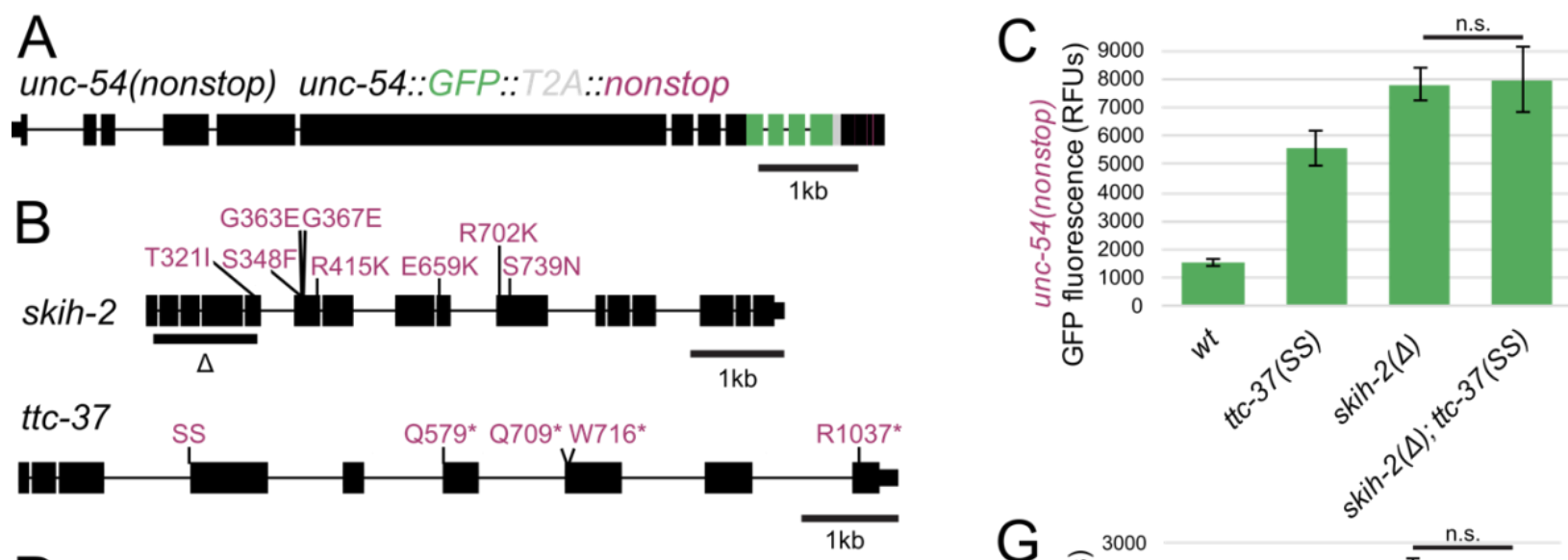

$\mathrm{D}_{\text {unc-54(rareArg) unc-54::T2A:::FLAG:::rareArg::GFP }}$

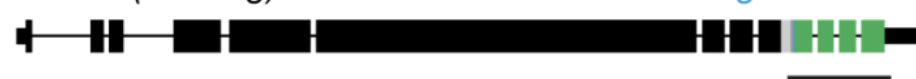

E

$1 \mathrm{~kb}$
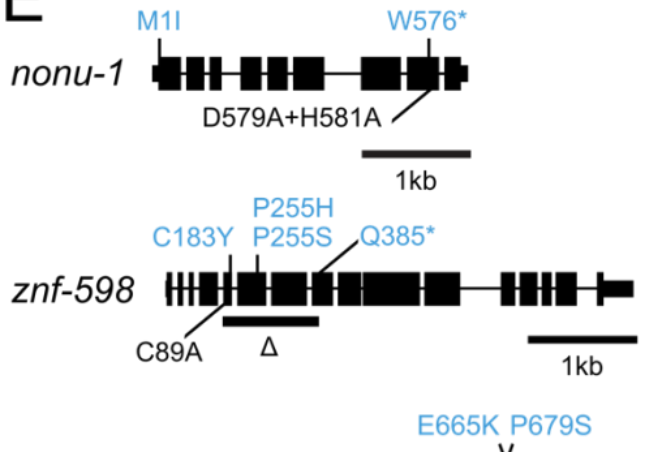

uba-1

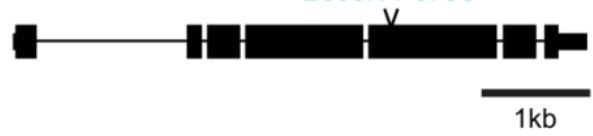

F

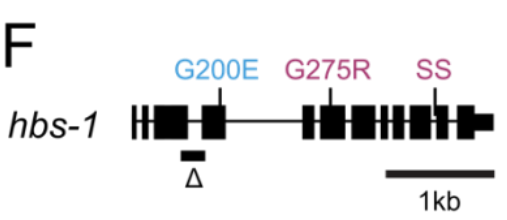

$1 \mathrm{~kb}$
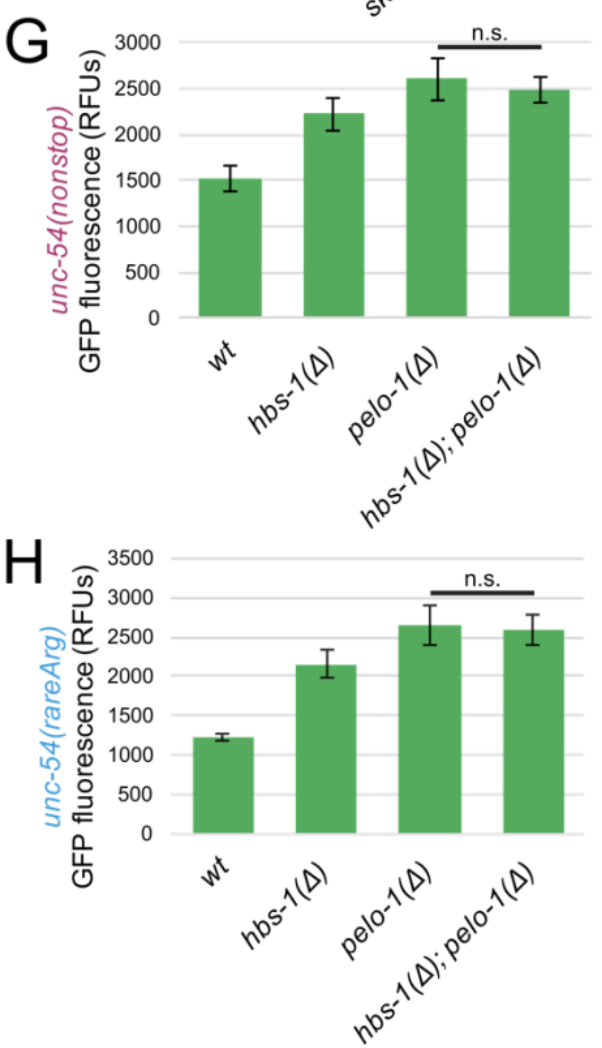

Figure 1. Nonstop and No-Go mRNA Decay suppressors identified via genetic screens. (A) Gene diagram showing annotated exons (black rectangles) of unc-54(nonstop). Colored rectangles represent CRISPR/Cas9 insertions at the endogenous unc-54 locus: GFP (green), T2A sequence (grey), and no stop codons (burgundy). (B) Diagrams of skih-2 and ttc-37 alleles. Black rectangles represent exons, thicker rectangles are CDS, and thin lines are introns. Mutations made via EMS in the Nonstop mRNA Decay screen (burgundy) and via CRISPR/Cas9 are above and below the gene, respectively. (C) Mean relative fluorescence units (RFUs) of indicated strains in the unc54(nonstop) background. One standard deviation shown as error bars. p values from Student's t-test. (D) Gene diagram showing annotated exons (black rectangles) of unc-54(rareArg). Colored rectangles represent CRISPR/Cas9 insertions at the endogenous unc-54 locus: T2A sequence (grey), FLAG (dark grey), 12 rare arginine codons (blue), and GFP (green). (E) znf-598, nonu-1, and uba-1 alleles drawn as in part (B). Mutations made via EMS in the rareArg screens (blue) and via CRISPR/Cas9 are above and below the gene, respectively. (F) hbs-1 alleles drawn as in part (B). Nonstop EMS mutations (burgundy) and a rareArg EMS mutation (blue) are above the gene. A deletion made by CRISPR/Cas9 is below. (G) Mean RFUs of indicated strains in the unc-54(nonstop) background. (H) Mean RFUs of 
occur. Strains with the unc-54(rareArg) construct and functional No-Go mRNA Decay machinery showed impaired movement and exhibited very low levels of GFP, supporting a model where mRNAs from this locus are targeted by No-Go mRNA Decay.

To validate the unc-54(rareArg) reporter, we crossed it with alleles of two factors known to be required for No-Go mRNA Decay: znf-598 and nonu-1 (Brandman et al., 2012; Juszkiewicz \& Hegde, 2017; Sundaramoorthy et al., 2017; D'Orazio et al., 2019; Glover et al., 2020). In each case we observed de-repression of the reporter, manifest as increased fluorescence and an improvement in animal movement and egg laying. The nonu-1 result is consistent with our prior work showing that this stretch of twelve rare arginine codons confers nonu-1-dependent mRNA decay (Glover et al., 2020). Given that most of our mechanistic understanding of No-Go mRNA Decay comes from work in S. cer (Doma and Parker, 2006; Simms et al., 2017; D'Orazio et al., 2019), and that genetic screens in human K562 cells have failed to identify the NONU-1 homolog (Hickey et al., 2020), we reasoned a genetic screen in C. elegans would prove insightful and augment information gained from other systems. Using unc-54(rareArg), we performed two genetic screens (Methods). Here we report nine alleles in No-Go mRNA Decay machinery (Fig 1E, Fig 1F).

Eight alleles were split amongst three readily-identifiable No-Go mRNA Decay components: nonu-1, znf-598, and uba-1. We found two mutations in nonu-1, as would be expected based on the unc-54(rareArg) reporter validation. We isolated four mutations in znf-598, three of which were missense mutations. One mutation, $\mathrm{C} 183 \mathrm{Y}$, is within a region that matches the consensus for a $\mathrm{C} 2 \mathrm{H} 2$ zinc finger in $C$. elegans and $H$. sapiens, but is not conserved in $S$. cer. Two alleles at P255 (P255S, P255H) mutated the highly conserved GHP motif at the start of a $\mathrm{C} 2 \mathrm{H} 2$ zinc finger. We also identified two mutations in uba-1, the sole $\mathrm{E} 1$ ubiquitin-activating enzyme in $C$. elegans. Both uba-1 mutations (E665K, P679S) exhibited poor viability, as would be expected as complete loss of $u b a-1$ is thought to be lethal (Kulkarni, et al., 2008). We confirmed the uba-1 result by crossing in a known temperature-sensitive loss-of-function allele of this gene (it129), and observed the expected temperature-dependent de-repression of GFP. As ZNF-598 is

208 known to function in other systems as a ubiquitin ligase (Juszkiewicz \& Hegde, 2017;

209 Sundaramoorthy et al., 2017), we expect that uba-1 loss-of-function compromised the Ub-

210 conjugation cascade, giving rise to unc-54(rareArg) de-repression. 

Among the factors hit in Nonstop and No-Go mRNA Decay screens was $h b s-1$, with two alleles from the Nonstop mRNA Decay screen and one allele from the No-Go mRNA Decay screen.

215 Requirement of $h b s-1$ in both pathways was confirmed by crossing in a deletion allele 216 constructed by CRISPR/Cas9. Interestingly, two mutations from the screens hit GTP-binding 217 and active site residues (G200E in No-Go mRNA Decay, G275R in Nonstop mRNA Decay), 218 consistent with a functional requirement for GTP binding and hydrolysis by HBS-1 in both 219 pathways. As HBS-1 homologs are known to function in a complex with PELO-1 homologs, we 220 also tested whether pelo-1 is required for both pathways. In hbs-1 and pelo-1 mutants, we observed consistent de-repression of unc-54(nonstop), and a similar pattern in unc-54(rareArg). Interestingly, in both reporters the effect of $h b s-1$ was slightly less than the effect of pelo-1, suggesting PELO-1 may have some residual activity in the absence of HBS-1, as might be expected given PELO-1's direct role in A-site recognition (Pisareva et al., 2011; Shoemaker \& Green 2011). In agreement with the idea that PELO-1 and HBS-1 function together, the double mutants (hbs-1 pelo-1) for both reporters were similar to the pelo-1 mutant (Fig 1G, Fig 1H).

\section{Exosome divergence at the Ski7 interface in C. elegans}

Despite the success of our genetic screens, homologs of two conserved SKI complex components (Ski7 and Ski8) remain unidentified in C. elegans. Sequence homology searches using either $S$. cer Ski8 or $H$. sap WDR61 identified six homologs in C. elegans (wdr-5.1, wdr5.2, wdr-5.3, lis-1, pfs-2, sel-10). All but PFS-2 exhibited conservation of residues known to be important for the S. cer Ski2-Ski8 interface (HxD, REF Halbach et al. 2013 23953113). Thus, genetic redundancy seems a plausible explanation for the lack of loss-of-function mutations in Ski8 homologs from the screen.

We also attempted to understand why a Ski7 homolog was not identified in our screen. Ski7 proteins exhibit little sequence conservation outside of a short "S3 motif" FXX[P/A]SPD[D/E] conserved in all known Ski7 homologs (Fig 2) (Marshall et al., 2018). Prior work using this motif failed to identify a Ski7 homolog in C. elegans (Marshall et al., 2018). We also performed

241 sequence homology searches with both conventional and custom-built software (see Methods),

242 and failed to identify a Ski7 homolog with the S3 motif. Given this result, we conducted

243 additional homology searches using lesser conserved motifs from Ski7 homologs (see

244 Methods). While we did identify a single gene with $\mathrm{N}$-terminus identical to some Ski7 homologs 
(M[A/S]RHR), a deletion spanning much of this gene failed to yield a phenotype in our unc-

We considered the possibility that Ski7 function may be encoded via alternative splicing of the hbs-1 gene as it is in several organisms; however, neither of the $h b s-1$ isoforms contain an S3motif. We looked for additional $h b s-1$ isoforms by analyzing RNA-seq and nanopore sequencing (Roach et al., 2020), but failed to identify anything more than the two annotated $h b s-1$ isoforms.

B

$$
\begin{aligned}
& \text { Scer ...ENFNRPSPDDIIQ... } \\
& \text { Spom ...LGFNAPSPDDIVL... } \\
& \text { Atha ...FKFDAPSPDDLVS... } \\
& \text { Agam ...FRFDVPSPDDVVL... } \\
& \text { Dmel ...FRFDTPSPDELVK... } \\
& \text { Hsap ...FDFKSASPDDIVK... } \\
& \text { Drer ...FSFNTPSPDDIVK... } \\
& \text { * : ***:: : }
\end{aligned}
$$

Figure 2. Exosome divergence at the Ski7 interface in C. elegans. (A) Structure of Ski7 (teal) bound to Csl4 (grey), focused on the S3 motif of Ski7. Structure from S. cerevisiae (Kowalinski et al., 2016). Dotted line indicates region of focus on right. Residues are colored to facilitate comparison with multiple sequence alignment, and numbered per S. cer. (B) Multiple sequence alignment of Ski7 and Csl4 proteins from several species, highlighting conservation of residues at the Ski7-Csl4 interface. Note conservation and divergence of some sequences in $C$. elegans; see text for further details. Species are as follows: Cele (Caenorhabditis elegans), Scer (Saccharomyces cerevisiae), Spom (Schizosaccharomyces pombe), Atha (Arabidopsis thaliana), Agam (Anopheles gambiae), Dmel (Drosophila melanogaster), Hsap (Homo sapiens), Drer (Danio rerio). Conservation below alignments is as follows: asterisks indicate identity, colons indicate amino acids with strongly similar properties, periods indicate amino acids with weakly similar properties. 
We sought to reconcile the apparent absence of a Ski7 sequence homolog in C. elegans despite an active Nonstop mRNA Decay pathway. The S3 motif is required for interaction between Ski7 and the exosome subunit (Cs/4) and also Nonstop mRNA Decay function (Fig 2A, REF Kowalinski et al., 2016 27345150; van Hoof et al., 2000 11027292). We thus examined the sequence of Csl4 homologs across eukarya. In virtually all species examined, the residues of Csl4 that interact with Ski7 are conserved, with the exception of $C$. elegans and related invertebrates (Fig 2B). Given the divergence of the exosome interface with which the Ski7 S3 motif interacts, there is no expectation that the $\mathrm{S} 3$ motif would be conserved. The remainder of the Ski7-exosome interface is composed of hydrophobic interactions and exhibits no recognizable sequence conservation (Kalisiak et al., 2017; Kowalinski et al., 2016). While this logic does explain the absence of a Ski7 sequence homolog, it does not explain why our genetic screen did not identify a Ski7 functional homolog (see Discussion).

mRNA decay is a major downstream consequence of ribosomal ubiquitination by ZNF598

In the No-Go mRNA Decay screen, we recovered several alleles of znf-598. ZNF-598 homologs are known to recognize ribosomal collisions and ubiquitinate sites on the small subunit, including RPS-10 (eS10) and RPS-20 (uS10) (Juszkiewicz \& Hegde, 2017; Sundaramoothy et al., 2017). By multiple sequence alignment, we identified a catalytic Cys (C89) (Sundaramoorthy et al., 2017) known to be required for Ub conjugation to substrates of Hel2/ZNF598 (Fig 3A), and generated a point mutation (C89A) via CRISPR/Cas9. In an immunoblot, we observed a shift to the expected size for Ub-RPS-10 which depended on the catalytic activity of ZNF-598 (Fig 3B). The znf-598(C89A) mutant phenocopied the deletion of znf-598 (Fig 3C), consistent with the idea that ZNF-598 requires its ubiquitin ligase domain for No-Go mRNA Decay repression. Taken together, these results support ZNF-598 repressing No-Go mRNAs via ribosomal ubiquitination in $C$. elegans, as is thought to occur in other systems.

Prior work has underscored the importance of ribosomal ubiquitination events in No-Go mRNA Decay. However, the precise functional contributions of individual ubiquitination sites has remained unclear, in part due to the difficulty in obtaining viable mutants in the relevant ribosomal proteins. Our system (C. elegans) seemed well-suited to address this question. We therefore generated single and double-mutant combinations of znf-598 along with its targets, then measured the effect on the unc-54(rareArg) reporter. Mutants of the ubiquitination sites on RPS-10 and RPS-20 exhibited a mild de-repression of the reporter, consistent with both sites 
contributing to stalling-induced repression (Fig S3, Fig 3D). Simultaneous knockout of ubiquitination sites on RPS-10 and RPS-20 generated a comparable de-repression to that observed in the znf-598 mutant. Furthermore, an rps-10 znf-598 double mutant phenocopied the de-repression observed in the znf-598 single mutant. Based on these results, we conclude that ZNF-598 must ubiquitinate at least RPS-10 on the ribosome, and RPS-10 and RPS-20 must both be ubiquitinated to elicit full repression during No-Go mRNA Decay.

Having established a requirement for ubiquitination by ZNF-598 in this system, we decided to investigate the relationship of ubiquitination to mRNA decay. A key effector of No-Go mRNA Decay is NONU-1, an endonuclease with Ub-binding domains identified in our screen (Fig 1E), which was also identified in our prior Nonstop mRNA Decay screen (Glover et al., 2020). We hypothesized that ZNF-598 deposits ubiquitin on stalled ribosomes during No-Go mRNA Decay to recruit NONU-1 and elicit mRNA cleavage. To test this model, we combined mutations in znf598 and its targets with nonu-1(AxA), a catalytic mutation of nonu- 1 that phenocopies a deletion in nonu-1 (Glover et al., 2020). A znf-598 nonu-1 double mutant phenocopied a znf-598 single mutant, which is in agreement with NONU-1 acting downstream of ubiquitination by ZNF-598 (Fig 3E). Interestingly, the magnitude of unc-54(rareArg) de-repression in a nonu-1 single mutant differed from that observed in either rps-10 or rps-20 mutant. Moreover, an rps-10 nonu1 double exhibited greater reporter de-repression than either single mutant. Taken together, these results indicate that NONU-1 functions downstream of ZNF-598 in No-Go mRNA Decay, though does not strictly rely on either RPS-10 or RPS-20 ubiquitination. Consistent with this model, we observed an increase of the unc-54(rareArg) reporter mRNA in znf-598, indicating that mRNA destabilization is a major downstream consequence of ZNF-598 (Fig 3F).

To determine if the relationship between ZNF-598 and NONU-1 is a broadly conserved phenomenon, we performed phylogenetic profiling. Briefly, phylogenetic profiling scores pairs of proteins using mutual information, which can be interpreted as the tendency of the protein pair to function together, either redundantly or sequentially. High mutual information can be achieved when two proteins are inherited together or lost together, or when two proteins are genetically

332 redundant and at least one protein is maintained (reviewed in Dey and Meyer 2015). We carried 333 out phylogenetic profiling by searching 111,921 profile HMMs (Mi et al., 2021) on genomic and 334 transcriptomic sequences from 473 protists as these represent diverse eukaryotic lifestyles (see 335 Methods). To validate this approach, we calculated mutual information between pairs of factors 336 known to function together in a complex, such as LTN-1/RQC-2 and PELO-1/HBS-1 (Fig S4) 


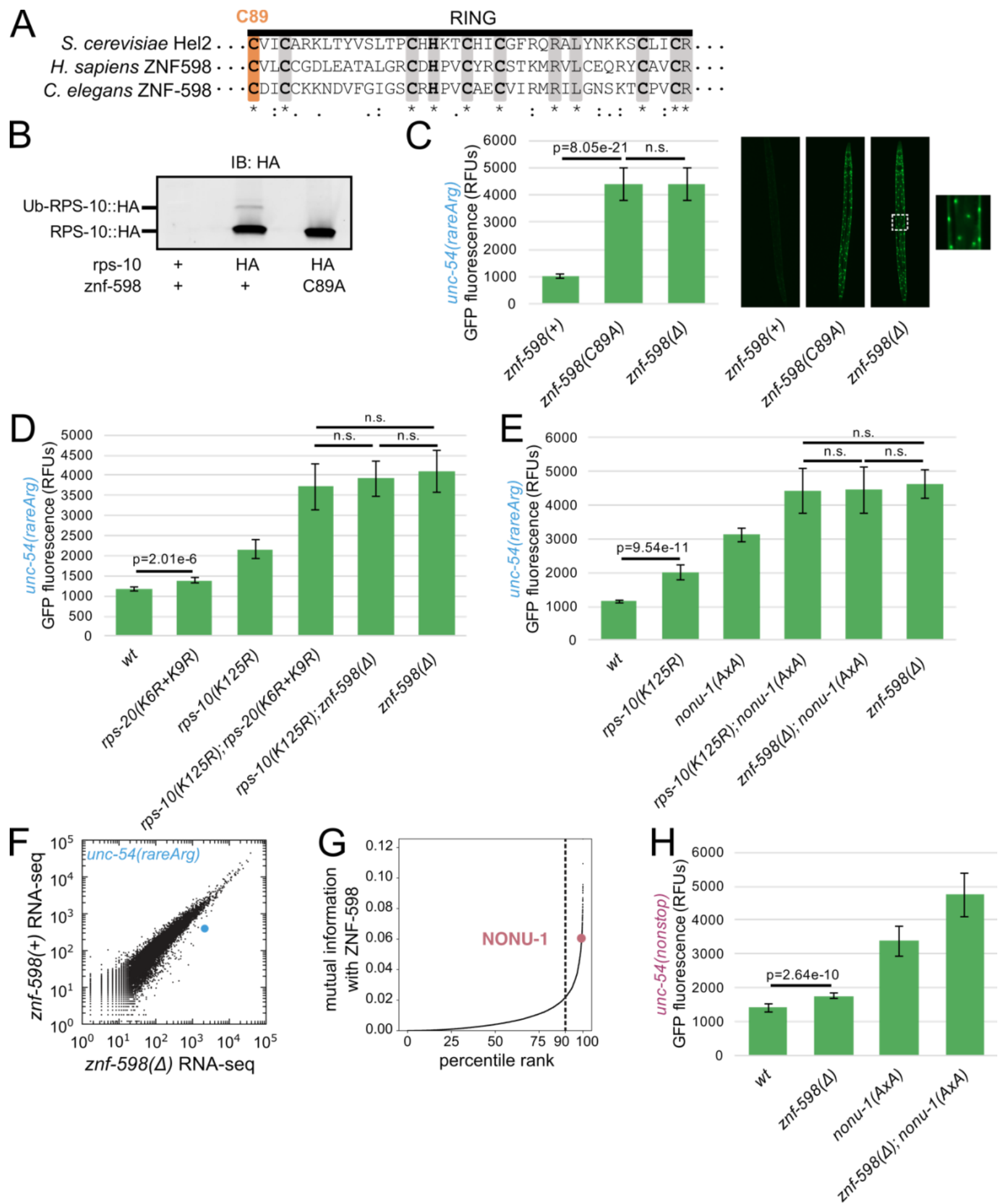

Figure 3. mRNA decay is a major downstream consequence of ribosomal ubiquitination by ZNF-598. (A)

Multiple sequence alignment of S. cer Hel2, H. sapiens ZNF598, and C. elegans ZNF-598 RING finger domain. Conserved residues are in grey and C89 in orange. Conservation is as shown in Fig 2B. (B) Western blot of indicated strains to monitor ubiquitination of HA-tagged RPS-10. (C) Mean RFUs of indicated strains in the unc-54(rareArg) background, with representative images at right. Zoom in shows nuclear localization of GFP. One standard deviation shown as error bars. $p$ values from Student's t-test. (D, E) Mean RFUs of indicated strains in the unc-54(rareArg) background. (F) Genome-wide RNA-seq of the indicated strains. All genes are shown as black dots and unc54(rareArg) in blue. (G) ZNF-598 mutual information plot. 90\% percentile cutoff is shown as a dashed line and NONU-1 is highlighted in pink. See Methods. (H) Mean RFUs of indicated strains in the unc-54(nonstop) background. 
347 (Kostova et al., 2017; Shoemaker et al., 2010; Becker et al., 2011; Hilal et al., 2016). LTN-

348 1/RQC-2 and PELO-1/HBS-1 exhibited high mutual information, scoring in each others' top

349 99.88\% (PELO-1 ranked 19th of 15,909 interactions with HBS-1) and 98.86\% (RQC-2 ranked

350 181th of 15,909 interactions with LTN-1), respectively. Similarly, we observed that ZNF-598 and

351 NONU-1 ranked in each others' top 99.38\% (NONU-1 ranked 99th of 15,909 interactions with

352 ZNF-598) (Fig 3G). Thus ZNF-598 and NONU-1 are a broadly conserved functional pair across

353 diverse eukaryotes, with our work in C. elegans suggesting that this pair functions in No-Go

354 mRNA Decay to degrade No-Go mRNAs.

We were curious to determine whether nonu-1 function depends on znf-598 in Nonstop mRNA Decay as well. Thus, we quantified their effects on the unc-54(nonstop) reporter (Fig $3 \mathrm{H}$ ). In contrast to its strong effect on the unc-54(rareArg) reporter, the znf-598 mutant showed mild unc-54(nonstop) de-repression. These results are consistent with a more modest role for znf598 in Nonstop mRNA Decay than that observed at unc-54(rareArg), and explains why our Nonstop mRNA Decay screens failed to identify znf-598 (Arribere and Fire, 2018; Glover et al., 2020). Surprisingly, nonu-1 exhibited a greater de-repression of unc-54(nonstop) than znf-598, and the double mutant expressed higher levels of GFP than either single mutant. The fact that nonu-1 and znf-598 together exhibit additive effects suggests that NONU-1 is recruited via an E3 ubiquitin ligase other than ZNF-598 during Nonstop mRNA Decay.

\section{Structural, computational, and functional evidence for ubiquitin-binding by HBS-1} involved in Ub-dependent processes, so we were initially surprised that this screen also identified the ribosome rescue factor $h b s-1$. We therefore examined HBS-1 to determine whether it could conceivably function in a Ub-dependent manner. domain, and two beta-barrel domains (Becker et al., 2011; Hilal et al., 2016). We noticed that

375 the N-terminal domain of HBS-1, while poorly conserved at the sequence level, adopts a distinct 376 triple helix fold that is classified by Pfam as a member of the ubiquitin-binding Uba clan (Fig 4A). 377 Notable members of the Uba clan include the Uba and Cue domains, found in S. cer Rad23 and 378 C. elegans NONU-1, respectively. In light of this structural homology, we scrutinized the 379 arrangement of surface-exposed residues in the $\mathrm{S}$. cer Hbs1 $\mathrm{N}$-terminus and noticed similar 380 amino acids at sites known to be important for the Uba-Ub interaction. Ub-binding domains are 
A

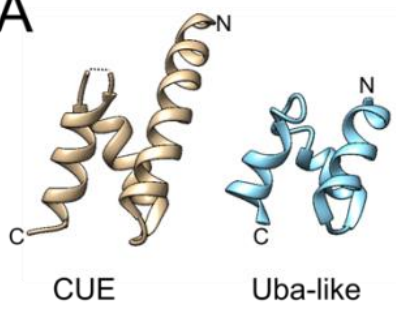

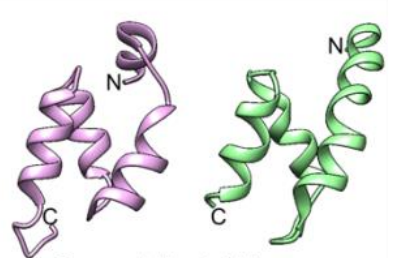

S. cer Hbs1 N-term

B

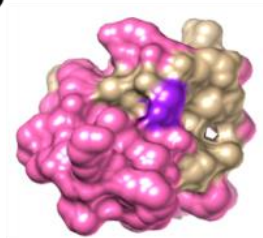

S. cer Hbs1 $\mathrm{N}$-term

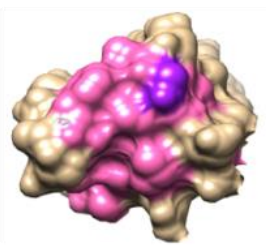

S. cer Rad23 Uba
C

Np14 ... AMWACQHCTFMNQ--PGTGHCEMCSL . . .

Atri ...GYWRCAICTYDNDNDDNLTSCDICGA...

Mbal ...GMWQCSICTYDND--ETSFCCDICGA...

Atha ...GLWRCAICTYDNV--ETMFVCDICGV...

Smoe ...PIWACPICTYDNL--EEHQSCEMCGV ...

Ppat ...GLWACPVCTFDNS--LDSLTCDICDT...

$$
\text { ** } * *: * \quad *:{ }^{*} \text {. }
$$
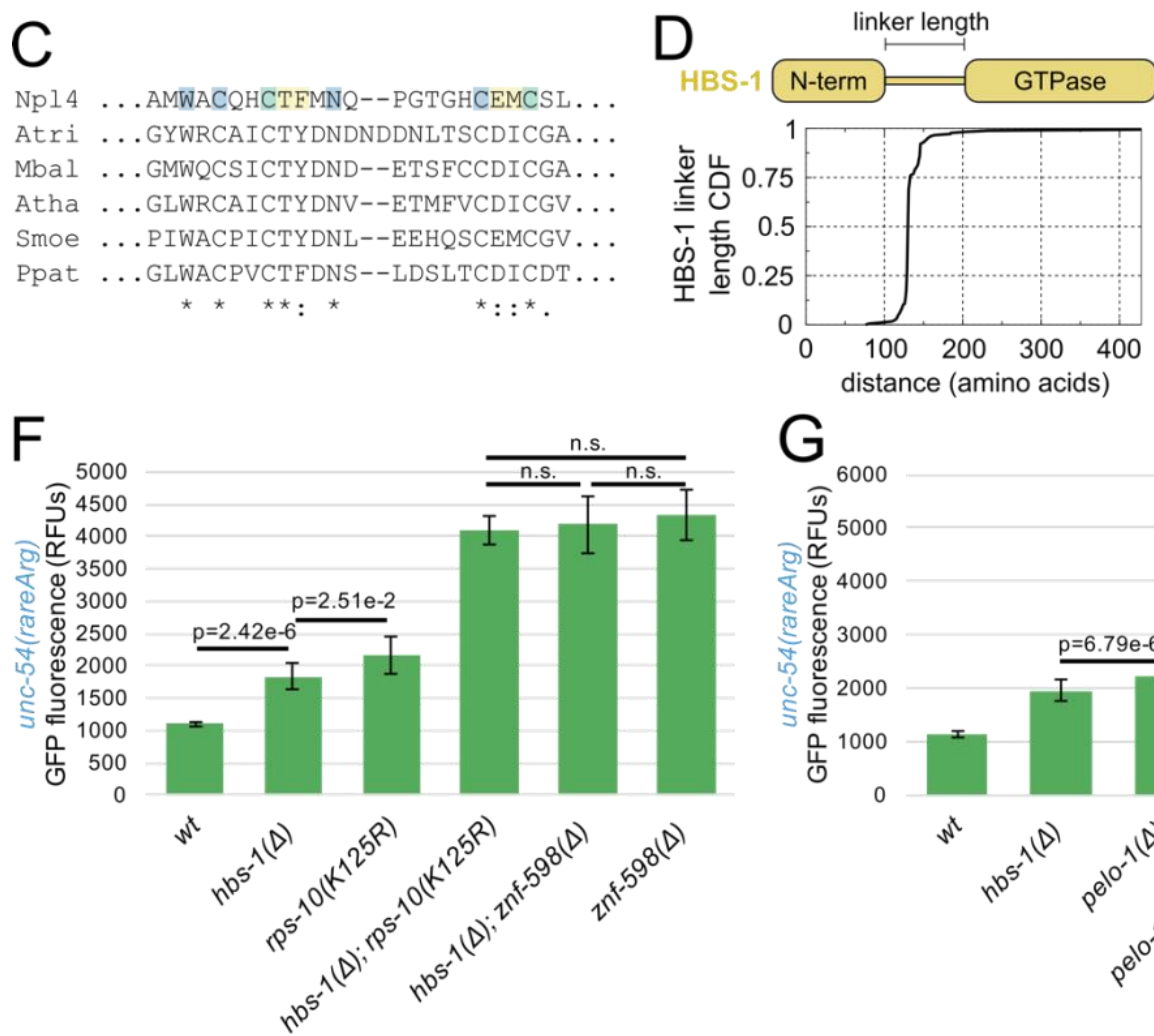

G

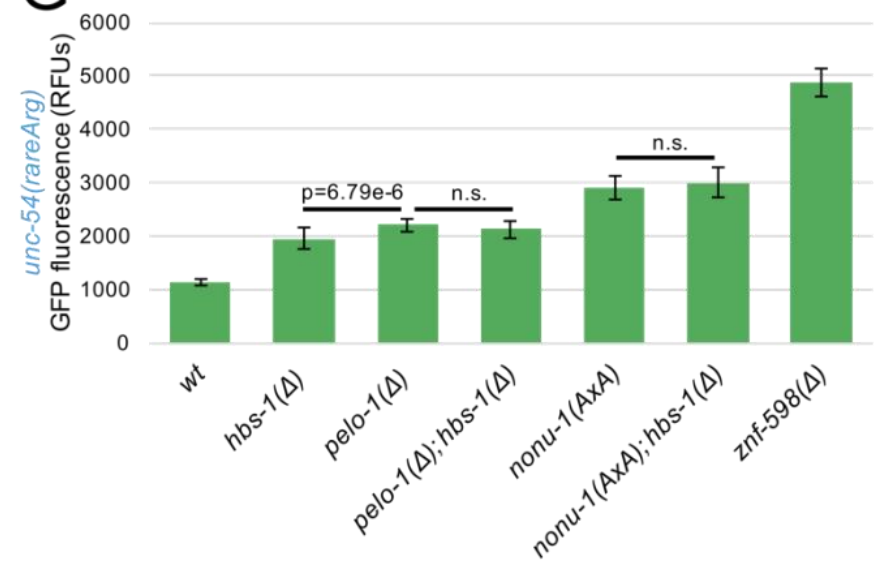

Figure 4. Structural, computational, and functional evidence for ubiquitin-binding by HBS-1. (A) Structural homology between $\mathrm{Hbs} 1 \mathrm{~N}$-terminal domain and ubiquitin-binding domains. Shown are structures representative of the UBA clan: CUE (tan, S. cer Vps9p, 1P3Q, [Prag et al., 2003]) and Uba-like (blue, S. cer Dcn1, 2L4E,

[Burschowsky et al., unpublished]). At right are two structures of the Hbs1 N-terminus from (Becker et al., 2011) (pink, 3IZQ) and (Hilal et al., 2016) (green, 5M1J). Amino and carboxy termini indicated with $\mathrm{N}$ and C, respectively. Note overall similarity in topology and fold across all four structures. (B) Structural surface depiction of the S. cer Hbs $1 \mathrm{~N}-$ terminal domain (left, 3IZQ) and Rad23 Uba domain (right, 2QSF). Three alpha helices are aligned in the same orientation, consisting of amino acids 20-83 of $\mathrm{Hbs} 1$ and 354-395 of Rad23. Hydrophobic residues are highlighted in pink, glycine in purple, and other residues in beige. (C) The N-terminal ZnF of plant $\mathrm{Hbs} 1$ is homologous to the Ubbinding $\mathrm{ZnF}$ of rat Npl4. Multiple sequence alignment of the Zinc Finger (ZnF) of Hbs1 from phylogenetically diverse plants. Residues that are highly conserved among Npl4 homologs are in blue, residues that contact $\mathrm{Ub}$ are in yellow, and residues that are both conserved and contact $\mathrm{Ub}$ are in green. Coloring and annotation of Npl4 residues from (Alam et al., 2004). Species are Atri (Amborella trichopoda), Mbal (Musa balbisiana), Atha (Arabidopsis thaliana), Smoe (Selganiella moellendorffii), and Ppat (Physcomitrium patens). (D) Top: Diagram of HBS-1 domain architecture. Bottom: Cumulative distribution function (CDF) of HBS-1 linker length in 177 orthologs of human HBS1L. Values calculated by subtracting the 3 ' end of the HBS-1 N-terminus domain from the 5 ' end of the GTPase domain. Domain bounds were determined by pfam and Hmmer. One data point was cropped from the graph along the $\mathrm{X}$-axis with a linker length of 428 amino acids. See Methods. (E) ZNF-598 mutual information plot. $90 \%$ percentile cutoff is shown as a dashed line and HBS-1 is highlighted in yellow. (F, G) Mean RFUs of indicated strains in the unc-54(rareArg) 
notable in their absence of substantial sequence identity (reviewed in Randles and Walters, 2012), an emergent property of the hydrophobic interface with Ub, providing an explanation for the low sequence identity among HBS-1 N-termini. Despite low sequence conservation, prior work identified a glycine surrounded by surface-oriented hydrophobic residues as a unique 407 characteristic of Uba domains (Mueller and Feigon, 2002). This feature is shared with the Hbs1 408 N-terminus domain, supporting a Ub-binding role for HBS-1 N-termini (Fig 4B). We also noticed 409 that plant HBS-1 homologs contain a conserved C4-type zinc finger homologous to a known Ub410 binding domain from rat (Fig 4C) (Alam et al., 2004). Thus, we hypothesize that the Ub-binding 411 function of HBS-1 emerged relatively early in the evolution of this factor. Taken together, these 412 observations support the idea that HBS-1's N-terminus binds ubiquitin, and that this feature is 413 broadly conserved across species.

415 To understand the role that Ub-binding might play in HBS-1 function, we analyzed cryo-EM 416 structures of yeast HBS-1 bound to ribosomes stalled on Nonstop and No-Go mRNA Decay 417 reporters. In both structures, the HBS-1 N-terminus is bound to the 40S subunit near uS10 and 418 uS3 (Hilal et al., 2016; Becker et al., 2011). uS10 is an established target of ZNF598/Hel2 in 419 both mammals and yeast, while uS3 is a substrate for ubiquitination in yeast (Juszkiewicz \& 420 Hegde, 2017; Sundaramoorthy et al., 2017, Ikeuchi et al., 2019). We hypothesized that the 421 HBS-1 N-terminus recruits the protein to stalled, ubiquitinated ribosomes, thereby allowing the 422 GTPase domain to carry out ribosome rescue.

As an initial evaluation of our model, we carried out two computational analyses. First, if HBS-1 needs to bind the ubiquitination sites on the 40S subunit and PELO-1 simultaneously, we reasoned there would be a conserved minimum linker length connecting the $\mathrm{N}$-terminus domain and the GTPase domain. Consistent with this, an analysis of $177 \mathrm{HBS}-1$ homologs identified a conserved length of 130 amino acids, with no homologs below a linker length of 76 amino acids (see Methods) (Fig 4D). This is an ample linker to span the distance from the ubiquitination sites near the mRNA entry tunnel to the A-site $(\sim 100 \AA, \sim 50$ amino acids, measured from Hbs1-bound ribosome structure 5M1J). Second, we examined the coevolution

432 of HBS-1 with ZNF-598 homologs, and observed a relatively high level of mutual information 433 suggesting that HBS-1 and ZNF-598 function together throughout eukarya (Fig 4E).

435 Our genetic analyses substantiated the notion that HBS-1 functions through ubiquitin binding. 436 Specifically, we observed znf-598 and nonu-1 are both epistatic to hbs-1 (Fig 4F, Fig 4G). 
437 Taken together, our structural, computational, and genetic observations support the idea that

438 ubiquitination is critical for ribosome rescue by HBS-1.

Deletion of ZNF-598 increases mRNA levels of and ribosomes on a No-Go mRNA Decay substrate

442 Given our genetic evidence placing znf-598 function upstream of both mRNA decay (via NONU-

443 1) and ribosome clearance (via HBS-1), we were curious to investigate the distribution of

444 ribosomes on unc-54(rareArg) with and without ZNF-598. To this end, we performed 28-30nt

445 monosome and 50-62nt disome Ribo-seq with and without znf-598 deletion (Ingolia et al., 2009;

446 Meydan et al., 2020; Han et al., 2020; Arpat et al., 2020; Zhao et al., 2021) (Fig 5, Fig S5).

447 Consistent with the stabilization of unc-54(rareArg) seen by RNA-seq, we observed an increase

448 in both monosome and disome footprints on the reporter in znf-598( $\Delta$ ) (Fig 5A). Within each

449 background, we found that monosome footprints were in agreement with disome footprints on

450 the reporter, indicating that monosome footprint count is a reasonable predictor of the

451 abundance in the captured disomes (Fig 5B).

453 We sought to determine whether the increase in ribosome footprints in znf-598( $\Delta$ ) was simply

454 the result of increased mRNA levels or whether there were additional znf-598-dependent effects

455 on ribosome footprint counts. We calculated the change in ribosome footprints per RNA-seq

456 reads between wildtype and $z n f-598(\Delta)$ and observed strong agreement between mRNA levels

457 and monosome density on unc-54(rareArg) (Fig 5C, Fig 5D). In accordance with the similar

458 amounts of monosome and disome footprints, we observed similar agreement between mRNA

459 levels and disome density. These data indicate that the increase in ribosomes on unc-

46054 (rareArg) in znf-598( $\Delta$ ) is largely explained by the increase in mRNA levels. Interestingly, we

461 note that monosome footprints in wildtype and znf-598( $\Delta)$ were enriched in the 5' end of the

462 mRNA (Fig 5E, Fig S6). This result could emerge from increased ribosome loading on No-Go

463 mRNAs, ribosome drop-off during elongation, or both.

We next drew our attention to the distribution of ribosomes along our reporter. In considering

466 ribosome position, it is important to understand that--while affected by mRNA levels--unc-

46754 (rareArg) is primarily a readthrough reporter. Ribosomes are required to translocate through

468 the rareArg stretch, and their success in completing this feat allows for GFP expression.

469 Evidence of stall readthrough and post-T2A production of FLAG::polyArg::GFP is visible by the

470 nuclear localization of GFP when de-repressed (Fig 3C). This observation revealed our

471 serendipitous construction of an $\mathrm{N}$-terminal motif (a run of arginines) matching the sequence 

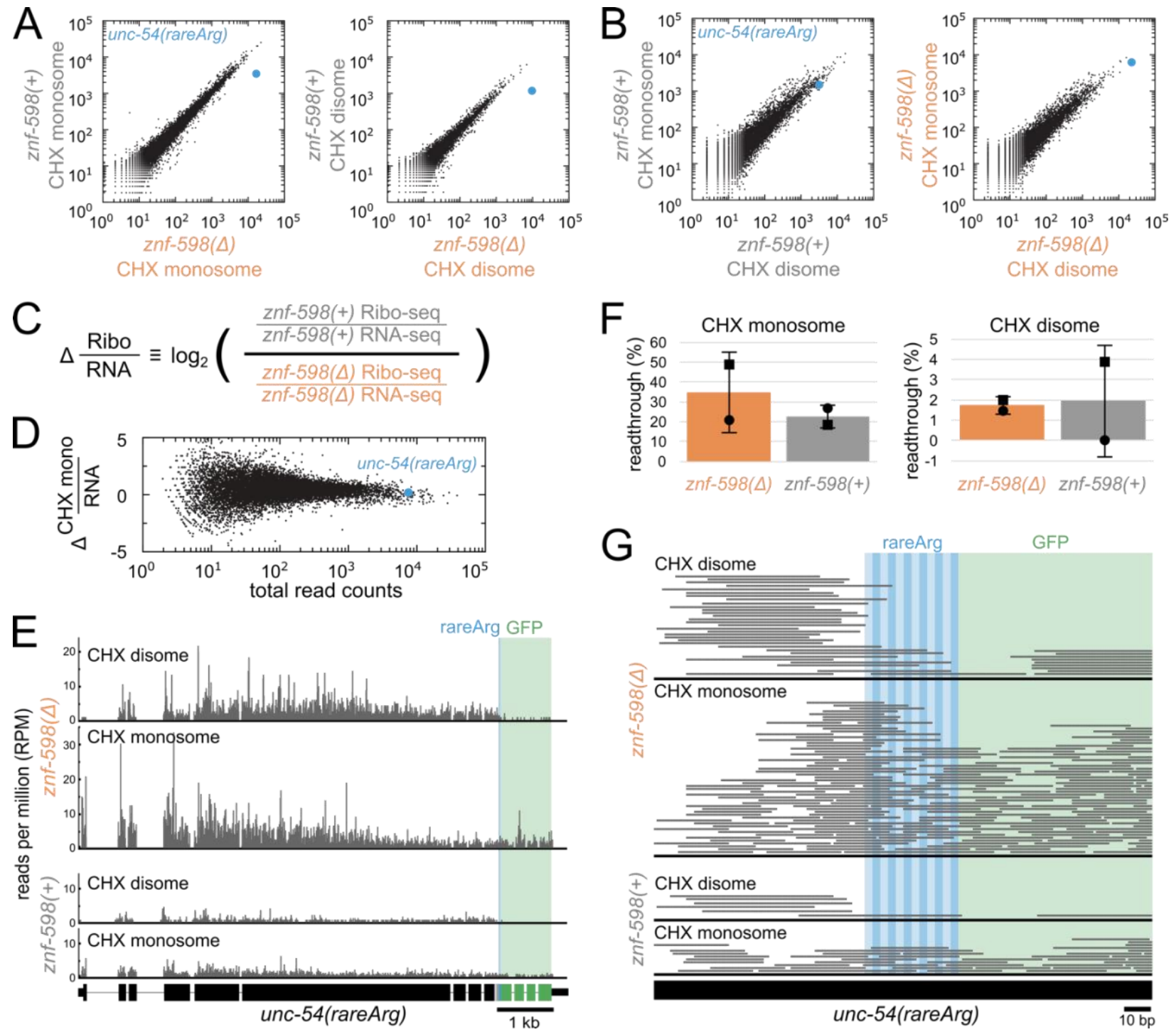

$$
\text { unc-54(rareArg) }
$$

Figure 5. Deletion of ZNF-598 increases mRNA levels of and ribosomes on a No-Go mRNA Decay substrate. (A) Rocket plots of $\mathrm{CHX}$ monosome and disome Ribo-seq reads per million (RPM) in znf-598(+) vs. znf-598(A). All genes are shown as black dots and unc-54(rareArg) is highlighted in blue. (B) As in (A), comparing CHX monosome vs. disome Ribo-seq RPM within one background. (C) Equation used to calculate the change in ribosome footprints per mRNA reads. Log change score is determined by dividing Ribo-seq read counts by RNA-seq read counts in each background, then dividing values for $z n f-598(+)$ by values for $z n f-598(\Delta)$. (D) Dot plot showing the change in ribosome footprints per mRNA reads between znf-598(+) and znf-598( $\Delta) \mathrm{CHX}$ monosome libraries. Y-axis position is the change score as calculated by the equation in (C). A more positive score indicates fewer monosome Ribo-seq read counts normalized to RNA-seq in znf-598( $\Delta$ ); a more negative score indicates fewer monosome Ribo-seq read counts normalized to RNA-seq in znf-598(+). X-axis position is the total number of read counts in all libraries. All genes are shown as black dots and unc-54(rareArg) is highlighted in blue. (E) Gene plot showing CHX disome (5062nt) and monosome (28-30nt) Ribo-seq RPM on unc-54(rareArg) in znf-598( $\Delta)$ and znf-598(+). Reads are aligned by their 5 ' ends. Locations of 12 rare arginine codons (blue) and GFP (green) are annotated. (F) Percentage of readthrough $\mathrm{CHX}$ monosome (28-30nt) footprints (left) and $\mathrm{CHX}$ disome (50-62nt) footprints (right) on unc54(rareArg) in indicated backgrounds from two biological replicates. Data points from replicate one are shown as black circles and replicate two are shown as black squares. One standard deviation shown as error bars. See Methods. (G) Pile up of CHX disome (50-62nt) and monosome (28-30nt) Ribo-seq reads around rareArg in the indicated backgrounds. Each arginine codon is represented by a vertical blue stripe. Each bar represents one read. Monosome datasets and disome datasets are subsampled to equal depth across the two backgrounds. 
requirements for a nuclear localization signal (Lu et al., 2021; Davis et al., 2021).

Given that GFP production from unc-54(rareArg) relies on readthrough, we hypothesized that some population of ribosomes must escape the stall and evade clearance. Indeed, we observed that monosome density downstream of the stall was $18-49 \%$ the level seen prior to the stall, with at most a mild effect by $z n f-598(\Delta)$ on this metric (Fig $5 F)$. The reduction in footprints downstream of the stall persisted in disomes (Fig 5F). We conclude that ZNF-598 does not dramatically alter the relative number of ribosomes upstream versus downstream of the stall.

Prior work in certain yeast mutants have observed frameshifting on No-Go mRNAs (Wang et al., 2018; Simms et al., 2019). We considered frameshifting as a mechanism by which ribosomes may pass through the arginine stall. Within and downstream of the stall, we detected no significant change in frame in ribosome footprints in either wild type or in znf-598( $\Delta$ ) animals, in agreement with yeast data on an arginine reporter (Fig S7A) (D'Orazio et al., 2019). As a second way of assessing the contributions of frameshifting to expression of unc-54(rareArg), we examined expression in a smg-1 mutant which has a nonfunctional Nonsense-mediated mRNA Decay pathway. Ribosomes that frameshift would be expected to terminate at an out-of-frame stop codon just past the arginine stall and trigger Nonsense-mediated mRNA Decay. We observed no increase in reporter expression in a smg-1 mutant (Fig S7B). Taken together,

514 these results argue against frameshifting as a substantial contributor to the expression of unc-

515 54(rareArg). We note these results are not surprising given that Mbf1 was intact in our

516 experiments; Mbf1 is known to enhance frame maintenance at stalls (Wang et al., 2018).

518 Considering the requirement of polyArg synthesis to produce GFP, we were surprised to see 519 few ribosomes decoding the rareArg stretch (Fig 5G). The low abundance of footprints in this 520 region was notably independent of mapping error; the arginine codons we selected were 521 arranged in a non-random order to enable unique read-mapping. In agreement with our results 522 on unc-54(rareArg), we observed a depletion of ribosomes decoding poly-basic sites genome523 wide, independent of a nucleotide capture bias (Fig S8, Fig S9). While in contrast to datasets

524 made in S. cer (Meydan et al., 2020; D'Orazio et al., 2019; Zhao et al. 2021), this observation is 525 consistent with multiple C. elegans datasets (Stadler and Fire, 2011; Glover et al., 2020), 526 human and zebrafish data (Han et al., 2020), and mouse data (Arpat et al., 2020) made using 527 the elongation inhibitor cycloheximide ( $\mathrm{CHX})$. 
In an effort to identify a stall-decoding species, we performed 18-30nt monosome Ribo-seq and 40-60nt disome Ribo-seq utilizing anisomycin (ANS) rather than cycloheximide (CHX) (Fig S10,

533 Methods). $\mathrm{CHX}$ inhibits translocation by binding the E-site and preventing deacylated tRNAs

534 from exiting the $\mathrm{P}$-site. Thus, $\mathrm{CHX}$ stabilizes ribosomes in a hybrid, pre-translocation state (Fig

$5356 \mathrm{~A})$. ANS inhibits peptide-bond formation by binding the A-site and competing with aminoacyl-

536 tRNAs for the peptidyl transferase center (reviewed in Dmitriev et al., 2020). Previous studies

537 have used ANS to stabilize ribosomes with short 21 nt footprints (Lareau et al., 2014). These

538 ribosomes are expected to be in a classical state with either an empty A-site (pre-

539 accomodation) or a free tRNA in their A-site (pre-peptidyl transfer) (Fig 6A). Given the

540 expectation for slow accommodation at rare codons, we investigated whether ANS-dependent

541 footprints resided in our arginine stretch.

Surprisingly, we captured both $21 \mathrm{nt}$ and $28 \mathrm{nt}$ monosome footprints via ANS, in contrast to ANS work done in yeast which captured very few 28nt footprints (Fig S10) (Lareau et al., 2014). We utilized this feature to probe the positions of multiple ribosome species on our reporter. Given the rarity of their cognate tRNAs, we expected the 12 rare arginine stretch to stall ribosomes with empty A-sites. However, we observed few ANS-stabilized monosome footprints (21nt or 28nt) within the stall, and even more footprints immediately following the stall (Fig 6B, Fig 6C). To further investigate this phenomenon, we considered the possibility that arginine-decoding ribosomes exist as disomes rather than monosomes.

To this end, we captured a wide range of in-frame disome species using ANS: 42-48nt, 51nt54nt, and 58-62nt (Fig 6A, Fig S10). We expect that the two smaller species lacking from $\mathrm{CHX}$ disome libraries represent events when both ribosomes are in the classical state (42-48nt), or one ribosome is in the classical state with the other in the hybrid state (51-54nt). Despite our capture of additional species via ANS, monosomes remained a general predictor of disome counts (Fig S11). Additionally, we observed a depletion of ribosomes within the 12 arginines on unc-54(rareArg) (Fig 6B, 6C). Consistent with these results on our reporter, we found a depletion of monosomes and disomes at arginine codons genome-wide (Fig 6D). Taken together, we propose that standard monosome and disome Ribo-seq protocols are unable to capture metazoan ribosomes at strong stalls. Future work will address the novel molecular features of these ribosomes and optimize their capture. 
A
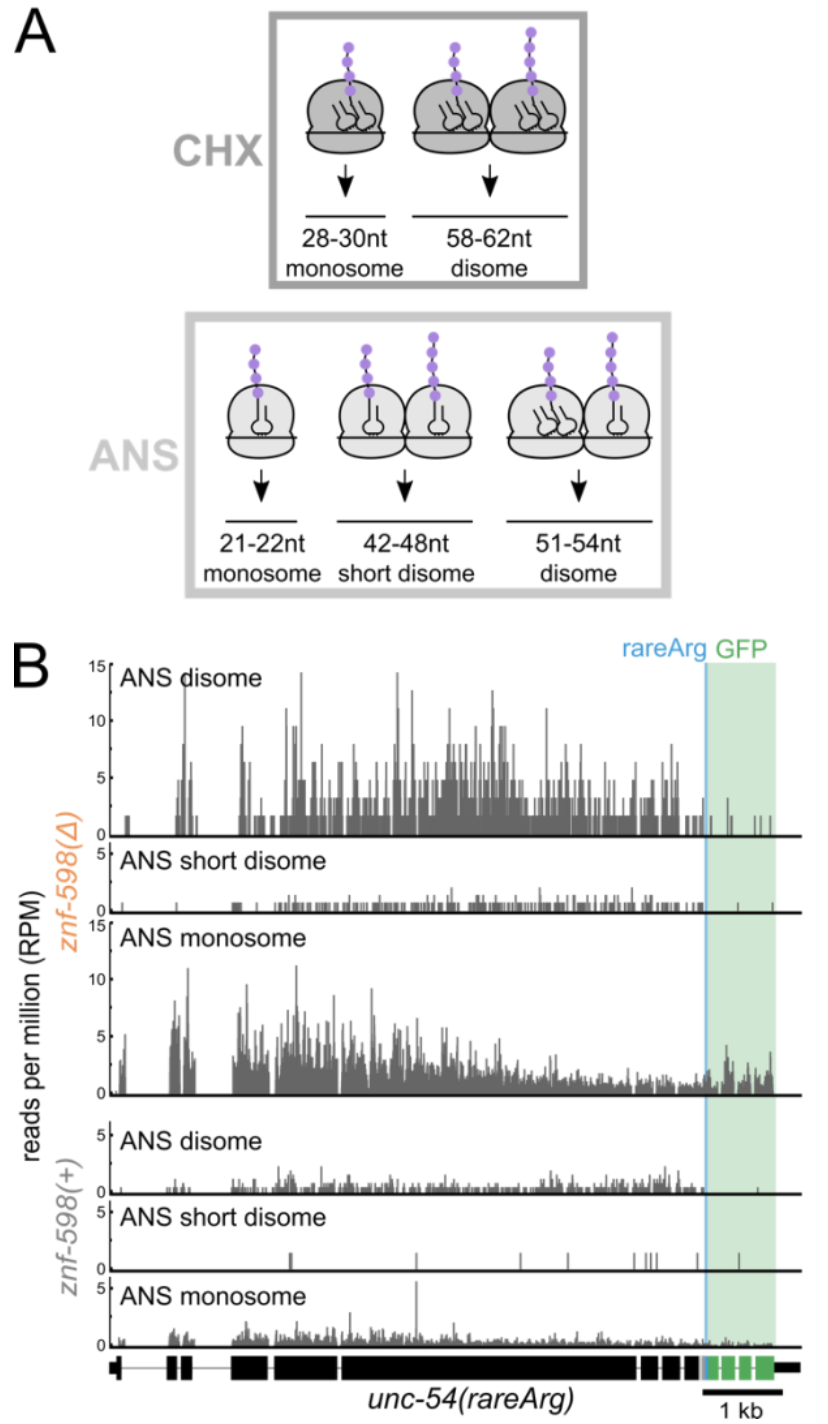
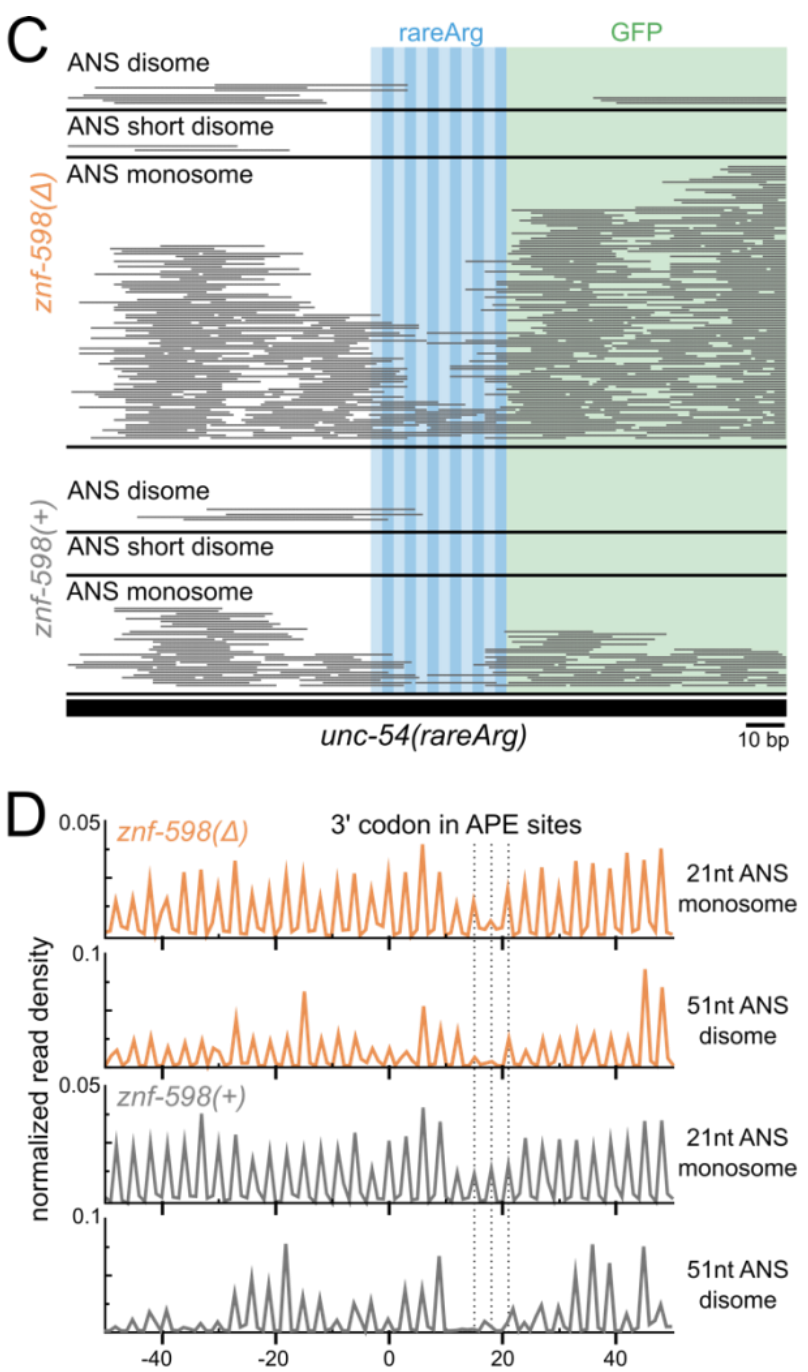

position of footprint 3 ' end relative to start of RRR (nt)

Figure 6. Ribo-seq libraries lack accumulations at arginine stalls. (A) Ribosome conformations and footprint sizes with cycloheximide vs. anisomycin. (B) Gene plot showing ANS disome (50-62nt) and monosome (18-30nt) Ribo-seq RPM on unc-54(rareArg) in znf-598( $\Delta$ ) and znf-598(+). Reads are aligned by their 5 ' ends. Locations of 12 rare arginine codons (blue) and GFP (green) are annotated. (C) Pile up of ANS disome (50-62nt) and monosome (18$30 \mathrm{nt})$ Ribo-seq reads around rareArg in the indicated backgrounds. Each arginine codon is represented by a vertical blue stripe. Each bar represents one read. Monosome datasets and disome datasets are subsampled to equal depth across the two backgrounds. (D) Normalized read density of ANS monosome (21nt) and disome (51nt) Ribo-seq reads genome-wide at RRR amino acid motifs in the indicated backgrounds. Reads are aligned by their 3' ends. Dotted lines indicate the footprint 3' end position when the third R of RRR is in each ribosomal site.

\section{DISCUSSION}

577 Here, we investigated the functions of and interplay between several factors required for

578 Nonstop and No-Go mRNA Decay. Through our Nonstop mRNA Decay system, we identified

579 functionally important residues in the SKI complex and suggested models consistent with

580 exosome-independent mRNA repression (Fig 1, Fig 2). By establishing No-Go mRNA Decay in C. elegans, we uncovered NONU-1 and HBS-1 recruitment as a downstream consequence of ribosomal ubiquitination via ZNF-598 (Fig 3, Fig 4). Subsequent work supported a novel Ub- 
583 binding function for the poorly characterized N-terminus of HBS-1. Additionally, our work

584 highlighted ZNF-598's critical role in destabilizing No-Go mRNAs and provided molecular

585 insights into the variety of ribosome species present at stall sites (Fig 5, Fig 6). Our analysis of

586 both Nonstop and No-Go mRNA Decay pathways revealed similarities in their dependencies

587 (pelo-1, hbs-1, nonu-1) and some key differences (znf-598).

588

\section{Architecture and function of the SKI complex in C. elegans}

590 Using both genetic screens and sequence homology searches, we have identified functional 591 components of the SKI complex including Ski2 and Ski3. Surprisingly, we did not uncover Ski7

592 nor Ski8 homologs in C. elegans. An approximate calculation suggests that our failure to find 593 Ski7 and Ski8 is not due to insufficient screening: between this and a prior study we recovered 59423 mutations in skih-2 and 7 mutations in ttc-37 (Fig 1B) (Arribere and Fire, 2018), yielding an 595 average hit rate of 6.05 and 2.09 mutations/kb, respectively. Using even the lower bound of 596 these two genes' mutation frequency, and given the size of yeast Ski8 ( 1.2kb) and human Ski7 597 ( 1.8kb), we would have expected at least one loss-of-function mutation in each protein $(92 \%$ 598 and $98 \%$ chance, respectively).

Ski8 is known to be an essential component of the heterotetrameric SKI complex in other systems (Ski2-Ski3-2xSki8). Our screens uncovered two mutations in Ski2 at G363E and G367E, residues that lie near the Ski8 interface, and we thus hypothesize that a Ski8 homolog exists in C. elegans (Fig S1). A simple homology search for Ski8 reveals six sequence homologs, suggesting that redundancy may have precluded identification of a functional Ski8. Alternatively, C. elegans' Ski8 may have essential functions outside of the SKI complex, as Ski8 is known to moonlight in meiotic recombination in yeast (Arora et al., 2004). Future biochemical analyses may identify which Ski8 homolog(s) function in the $C$. elegans SKI complex. candidate Ski8 homologs contain an unstructured N-terminal extension. We hypothesize that these homologs are fusions of Ski7 and Ski8 function into a single gene. Alternatively (and

612 intriguingly), it is also possible that the $C$. elegans SKI complex functions without a functional

613 Ski7, i.e., that $C$. elegans SKI does not need an association with the 3'>5' exosome to repress

614 its targets. This idea is supported by the observation that the SKI complex alone can extract

615 RNA from ribosomes (Zinoviev et al., 2020). Future work will hopefully clarify the composition 616 and functions of the SKI complex essential to Nonstop mRNA Decay. 
619 Our work supports a model where both NONU-1 and HBS-1 function downstream of ribosomal

620 ubiquitination by ZNF-598 in No-Go mRNA Decay, though neither effector strictly relies on RPS-

62110 ubiquitination (Fig 3, Fig 4). What precise ribosomal ubiquitination sites are then recognized

622 by NONU-1 and HBS-1? Existing work is consistent with ribosomal ubiquitination being a central

623 component of No-Go mRNA Decay and highlights the importance of RPS-10 and RPS-20;

624 however, the contributions of each ubiquitination site have remained unclear (Juszkiewicz \&

625 Hegde, 2017; Sundaramoorthy et al., 2017).

Here, we conclude that RPS-10 and RPS-20 must both be ubiquitinated to elicit full repression during No-Go mRNA Decay, and we showed that simultaneous knockout of their ubiquitination phenocopied znf-598. Our work shows that these sites can function as partially redundant recruitment routes for NONU-1 and HBS-1. Moreover, the level of de-repression in nonu-1 and hbs-1 differed from that observed in either rps-10 or rps-20. These data are consistent with a model where RPS-10 or RPS-20 ubiquitination do not act alone in effector recruitment. It is possible that RPS-10 and RPS-20 ubiquitination precede further ubiquitination at other ribosomal sites (Garzia and Meyer et al., 2021 34348161). Surprisingly, an rps-10 nonu-1 double and an rps-10 hbs-1 double exhibited greater de-repression than either single mutant. We hypothesize that in lieu of NONU-1 or HBS-1 function, RPS-10 ubiquitination recruits additional factors (such as another endonuclease) to elicit decay of No-Go mRNA Decay substrates (Glover et al., 2020; Navickas et al., 2020). Further genetic work will be required to elucidate the network of ubiquitination sites functioning in No-Go mRNA Decay.

642 Following our evidence of ZNF-598's critical role in No-Go mRNA Decay, we were surprised to 643 observe a minor and NONU-1-independent role for ZNF-598 in Nonstop mRNA Decay (Fig 3H).

644 We suspect NONU-1 is recruited to Nonstop mRNA Decay substrates by an E3 ubiquitin ligase 645 other than ZNF-598. In yeast and mammals, ZNF-598 is known to require at least two collided 646 ribosomes which form a distinct collision interface suitable for ubiquitination (Simms et al., 2017; 647 Juszkiewicz et al., 2018; Matsuo et al., 2020). Given that there are structural differences in 648 collided ribosome conformation during Nonstop mRNA Decay vs. No-Go mRNA Decay (Hilal et 649 al., 2016; Becker et al., 2011), we hypothesize that an E3 ligase other than ZNF-598 is better 650 equipped to recognize ribosomes stalled at the 3'end of an mRNA and trigger Nonstop mRNA 651 Decay. Other E3 ligases have been implicated in surveillance, such as Not4 and RNF10, and 
652 future work will hopefully clarify the recruitment mechanisms of each (Ikeuchi et al., 2019;

653 Garzia and Meyer et al., 2021).

655 Our Nonstop mRNA Decay reporter does, however, utilize ZNF-598 to some degree. The mild 656 Nonstop mRNA Decay de-repression seen in znf-598 could be interpreted to indicate that No-

657 Go mRNA Decay-inducing ribosomal collisions are a minority of stalled ribosomes during 658 Nonstop mRNA Decay. We hypothesize that these collisions are worsened in a nonu-1 mutant 659 and thus recruit ZNF-598 for clearance and decay. These ideas are consistent with other 660 models suggesting multiple types of collisions, with the relative amount of each collision 661 depending on the genetic background (Ikeuchi et al., 2019).

\section{The timing of ribosome rescue and a conserved ubiquitin-binding function for HBS-1} We have previously observed NONU-1 cleavage near the A-site of a collided ribosome (Glover et al., 2020) and we now propose a method for the rescue of this internally-cleaved ribosome (Fig 4G). Structural work positioning S. cer Dom34 in a stalled ribosome suggests a requirement for an mRNA-free A-site, as inferred by its beta-loop occluding the mRNA channel (Hilal et al., 2016), and we expect that ribosomes with cleavages in their A-sites would thus be substrates for PELO-1/HBS-1.

However, this model fails to explain how single mutants of $h b s-1$ and pelo- 1 have de-repression phenotypes in the unc-54(rareArg) reporter (Fig 1H, Fig 4G). Given a model where rescue is downstream of mRNA cleavage, how would deletion of a rescue factor that recognizes cleaved mRNA in a ribosome allow such a terminally-stalled ribosome to complete GFP? We speculate that HBS-1 and PELO-1 may function in an arrest pathway clearing some ribosomes independent of mRNA cleavage and that this pathway occurs on our reporter. PELO-1 could feasibly bind internally-stalled ribosomes independent of cleavage via A-site mRNA displacement as seen by the bacterial rescue factor ArfB (Carbone et al., 2020). An mRNAcleavage-independent mechanism would thus allow for translation of the downstream GFP on our reporter in pelo-1 and hbs-1 mutants. We hypothesize that the leading ribosome in a pileup of collided ribosomes may evade mRNA cleavage and is therefore poised to translate GFP in

682 the absence of rescue.

684 We also characterized a novel Ub-binding recruitment mechanism for HBS-1. Our functional, 685 computational, and structural results support a model in which HBS-1 function in No-Go mRNA 686 Decay depends on ribosomal ubiquitination (Fig 4). We therefore hypothesize that HBS-1 is 
687 targeted to a stall by its N-terminus after an E3 ligase ubiquitinates sites on the ribosome (e.g.,

688 RPS-10 and RPS-20), subsequently localizing PELO-1. Taken together, our findings suggest

689 that HBS-1 and PELO-1 have a route for locating stalled ribosomes via ribosomal ubiquitination

690 and could conceivably utilize this signal during an mRNA-cleavage-independent arrest pathway.

\section{Scenarios leading to missing arginine-stalled ribosomes}

693 Surprisingly, we observed a lack of a notable stalled ribosome peak in either monosomes or 694 disomes at the site on unc-54(rareArg). Instead, we observed a depletion of footprints at the 695 stall (Fig 5, Fig 6). While this result was contrary to the arginine-stalled ribosomal peaks seen in 696 yeast (Meydan et al., 2020; D’Orazio et al., 2019; Zhao et al. 2021), a subdued ribosomal 697 presence at arginine stretches has been previously reported in worms, zebrafish, mice, and 698 humans (Stadler and Fire, 2011; Glover et al., 2020; Han et al., 2020; Arpat et al., 2020). 699 Moreover, our genome-wide analysis at arginine and lysine stretches exhibited a similar 700 depletion (Fig 6D; Fig S8).

This result might be interpreted as faster decoding within arginine and lysine stretches; however, multiple lines of evidence argue against this model:

(1) Ribosomes stalled on our reporter are faced with two of the rarest codons in C. elegans. towards delayed aminoacyl-tRNA accommodation and therefore slow decoding at the stall site. (2) On our reporter and genome-wide, poly-basic stretches are expected to trigger slower translocation due to interactions between the positively-charged nascent peptide and the peptide exit tunnel (Lu and Deutsch, 2008; Chandrasekaran et al., 2019). We expect these

711 interactions to produce increased ribosomal density within the poly-basic-encoding codons and 712 immediately downstream.

713 (3) Ribosomes entering a strong stall could conceivably experience faster or discontinuous 714 elongation via a yet-unknown mechanism, akin to "shunting" observed during translation of 715 some viruses (Ryabova and Hohn, 2000). This model would be consistent with lower density at 716 the stall, but would also result in increased density after the stall due to a return to normal 717 elongation. We did not observe an accumulation of ribosomes post-stall on our reporter or 718 genome-wide, thus we do not have support for a faster elongation model. Furthermore, on our 719 reporter, "pushed" ribosomes would have to first produce at least some polyArg peptide to 720 generate the NLS::GFP as observed (Fig 3C). 
721 Given the lack of support for faster elongation through arginines and lysines, we propose

722 several non-mutually exclusive explanations for a missing arginine- and lysine-stalled species:

(1) Ribosomes stalled within the arginine stretch on unc-54(rareArg) may experience

725 preferential disassembly by a factor such as the ASC-1 complex (Juszkiewicz et al., 2020).

726 While in agreement with the reduced ribosomal density following the stall on unc-54(rareArg),

727 this model is not consistent with the reappearance observed at genome-wide arginines and

728 lysines. Furthermore, it remains unclear why mutations in these factors were not identified in our 729 screens.

730 (2) Ribosomes decoding strong stall sites may be present as a lowly-abundant, higher-order structure. This structure could consist of trisomes, tetrasomes, pentasomes, etc. which are difficult to visualize and capture via a sucrose gradient. Alternatively, this structure could be a ribosome (monosome or higher-order) with a tightly-bound accessory factor giving rise to a unique footprint size. We considered a broad range of footprint sizes in this work and did not observe this species associated with either monosomes or disomes.

(3) Stalled ribosomes that elicit No-Go mRNA Decay may be unstable in lysates. Ribo-seq is known to preferentially capture elongating ribosomes. If the stalled ribosome species was not easily trapped by translation elongation inhibitors (such as $\mathrm{CHX}$ and ANS), the stalled species may escape detection.

740 (4) No-Go mRNA Decay may occur in a subcellular compartment which is not captured in 741 standard cellular lysis conditions. Given evidence of specialized subcellular compartments for 742 translation and mRNA decay (such as stress granules and P-bodies) (reviewed in Das et al.,

743 2021), some proportion of No-Go mRNA Decay could feasibly occur in a compartment which is 744 lost during lysis.

Despite missing ribosomes at arginine and lysine stretches, we captured a robust signal of ribosomes paused at poly-proline stretches, specifically the Pro-Pro-Gly motif (Fig S8B). This

748 result is consistent with several metazoan disome Ribo-seq studies where proline stalls were 749 most abundant (Han et al., 2020; Arpat et al., 2020). The mechanism of proline stalling is 750 strikingly different from that which is expected during arginine and lysine stalling: peptidyl 751 transfer between proline and glycine proceeds more slowly than at other codons and is aided by 752 elF5A (Gutierrez et al., 2013; Schmidt et al., 2016; Schuller et al., 2017). Taken together, we 753 conclude that the ribosomal states including PPG-stalled ribosomes are distinct from those 754 states stalled on arginine and lysine stretches. 
In light of many recent disome studies, we propose a model where not all stalled ribosomes are captured in standard Ribo-seq preparations, and that existing metazoan disome data represent ribosomes in the PPG-like-stall state. Our work with a validated No-Go mRNA Decay reporter provides evidence that the PPG-like-stall state is distinct from that which occurs during No-Go mRNA Decay. In order to study endogenous targets of No-Go mRNA Decay in metazoans, future work will need to elucidate the unique features of arginine- and lysine-stalled ribosomes to optimize their capture. Identification of this species would therefore provide new molecular insights into decay-inducing ribosomal stalling vs. programmed or transient ribosomal pausing.

\section{ACKNOWLEDGEMENTS}

We thank all the members of the Arribere lab for feedback on the manuscript, thoughtful discussion, and technical support. We thank Ben Abrams and the University of California, Santa Cruz (UCSC) Microscopy Facility for assistance with imaging and quantification. This work was supported in part by a T32 Training Grant Fellowship from the National Institute of General Medical Sciences (NIGMS) (5T32GM133391-02) to P.C.M., a National Science Foundation (NSF) Graduate Research Fellowship (NSF DGE-1842400) to M.L.G., an R01 grant from the NIGMS (1R01GM131012-01) to J.A.A., a Searle Scholars award to J.A.A., and start-up funds from UCSC to J.A.A.

\section{AUTHOR CONTRIBUTIONS}

P.C.M. and J.A.A conceived the study and designed, conducted, and analyzed experiments. performed the HBS-1 linker and Uba-homology analyses. M.L.G. recovered and mapped Nonstop screen alleles, with some help from T.A.E. with genomic sequencing. N.V. mapped some Nonstop and No-Go screen alleles and performed mutual information analysis. The manuscript was written by P.C.M. and J.A.A, with some sections by A.L.P. and N.V. and input from all authors.

\section{COMPETING INTERESTS}

785 The authors declare no competing interests.

\section{DATA AND MATERIALS AVAILABILITY}

788 The data discussed in this publication have been deposited in NCBI's Gene Expression

789 Omnibus (Edgar et al., 2002) and are accessible through GEO Series accession number

790 GSE187348 (https://www.ncbi.nlm.nih.gov/geo/query/acc.cgi?acc=GSE187348). 


\section{SUPPLEMENTARY MATERIALS}

792 Table S1: C. elegans strains and oligos

793 Figures S1-S11

794

\section{METHODS}

\section{C. elegans strain construction and propagation}

C. elegans strains were derived from the N2 background (VC2010 [Thompson et al., 2013]), excluding the Hawaiian strain used for suppressor mapping. Animals were grown at $16 \mathrm{C}$ or $20 \mathrm{C}$ on NGM plates seeded with OP50 as a standard food source (Brenner, 1974). Some strains were obtained from the CGC, which is funded by NIH Office of Research Infrastructure Programs (P40 OD010440).

CRISPR/Cas9 was performed to introduce genomic edits as previously described (Arribere et identical phenotypes. Mutant combinations were generated by crossing. A full list of strains, sequences of mutant alleles, and sources is available in Table S1.

All worm strains were maintained at 20C. L4 animals were selected and anesthetized in $3 \mathrm{uL}$ EN50 with $1 \mathrm{mM}$ levamisole in a microscope well slide with a $0.15 \mathrm{~mm}$ coverslip. A Zeiss AxioZoom microscope was used with a 1.0x objective to acquire all images. The following parameters were used for all images: exposure time 250ms., shift 50\%, zoom 80\%. 15-25 representative animals were imaged for each strain. All comparisons shown are between

814 images obtained during the same imaging session.

We used FIJI to define the area of the animal, subtract the background, and determine mean pixel intensity for the area of each animal. A mean and standard deviation of fluorescence intensity was calculated for each strain based upon quantification of 15-25 representative images per strain. Student's t-test was used to determine $p$-values.

\section{EMS mutagenesis and suppressor screens}

822 EMS mutagenesis was performed in three independent screens, one using unc-54(cc4092)

823 (nonstop) (Glover et al., 2020) and two using unc-54(srf1004) (rareArg), essentially as

824 described (Arribere and Fire, 2018). 
826 Briefly, a large population of each strain was washed with M9 to a final volume of $4 \mathrm{~mL}$. EMS

827 was added to a final concentration of $\sim 1 \mathrm{mM}$ and animals were incubated at room temperature

828 for $4 \mathrm{hr}$ with nutation. Animals were washed and left overnight at room temperature on NGM

829 plates seeded with OP50. The next day, animals were washed and eggs were collected by

830 hypochlorite treatment. 100-200 eggs were placed on single small NGM+OP50 plates and

831 allowed to propagate. Plates were screened for F2/F3 animals with increased GFP fluorescence

832 and increased movement. Only a single isolate was kept per NGM plate to ensure

833 independence of mutations.

835 The first rareArg screen was saturated with hits at znf-598, occluding our ability to recover

836 additional alleles at other loci. To address this, we constructed a double-balanced strain

837 covering the region of chromosome II harboring znf-598, similarly to that done in an NMD

838 suppressor screen (Cali et al., 1999). This strain (WJA 1040) was homozygous on chromosome

839 I for unc-54(srf1004) and heterozygous on chromosome II for mnC1[dpy-10(e128) unc-52(e444)

840 nls190 let-?](AG226) and tra-2(e1095) (CB2754). Animals homozygous for mnC1 or

841 homozygous for tra-2(e1095) were inviable or Tra/sterile, respectively, allowing us to eliminate

842 recovery of recessive suppressors within the balanced region. This strain was subjected to EMS

843 mutagenesis as described above. Only isolates maintaining the $m n C 1 /$ tra-2 heterozygous

844 balancer were kept, as seen by myo-2::GFP, a nonTra phenotype, and subsequent viability.

\section{Suppressor mapping and identification}

847 Following recovery of mutants from EMS screens, we employed a Hawaiian SNP mapping

848 approach as described (Doitsidou et al., 2010). We crossed each isolated suppressor to

849 Hawaiian (CB4856) unc-54(cc4112) males (expressing an UNC-54::mCherry fusion engineered

850 by CRISPR/Cas9). Cross progeny were isolated and allowed to self-fertilize. The F2 GFP+

851 progeny were then backcrossed to the unc-54(cc4112) males at least 2 times.

After rounds of backcrossing, several phenotypically suppressed animals ( 20-30) for a given

854 mutant were pooled together onto a single plate and allowed to starve. Upon starvation, animals

855 were washed off the plate with $1 \mathrm{~mL}$ EN50, and further washed with EN50 to remove residual E.

856 coli. Genomic DNA was extracted after proteinase $\mathrm{K}$ treatment and resuspended in 50uL TE

857 pH7.4. 50ng genomic DNA was used as an input for Nextera (Tn5) sequencing library

858 preparation. Libraries were sequenced at the Vincent J. Coates Genomics Sequencing

859 Laboratory at UC Berkeley on a HiSeq 4000 (nonstop) and at Novogene Corporation Inc. UC

860 Davis Sequencing Center on a NovaSeq 6000 (rareArg). 
861 Reads were mapped to the $C$. elegans genome using bowtie2 (version 2.3.4.1). Reads were assigned using GATK (McKenna et al., 2010) and a previously published dataset of Hawaiian SNPs (Thompson et al., 2013). The fraction of reads that were assignable to Hawaiian or N2 animals was calculated across the genome, and linkage was identified by portions of the genome with $0 \%$ Hawaiian. Regions of linkage were then manually inspected to identify candidate lesions/loci.

\section{Immunoblotting}

Animals were propagated on NGM plates with OP50 until nearly starved, then washed twice with $1 \mathrm{ml} \mathrm{M9}$ and flash frozen in liquid nitrogen. Worm pellets were boiled for $10 \mathrm{~min}$ at $99 \mathrm{C}$ in $1 \mathrm{x}$ SDS loading buffer (0.5M Tris- $\mathrm{HCl}, 100 \%$ glycerol, 20\% SDS, 1M DTT, 5\% bromophenol blue). Samples were vortexed and spun to pellet animals and supernatants were collected. Protein was quantified by Qubit and 15ug protein was run on a 4-20\% Mini-PROTEAN(R) TGX StainFree Protein gel (Bio-Rad Laboratories, Inc.). Protein was transferred to a low background fluorescence PVDF membrane (Millipore) and the membrane was blocked in $1 \%$ nonfat milk in 1x TBST. Anti-HA antibody (Enzo Life Sciences 16B12) was used at a 1:2000 dilution to detect the HA-tagged RPS-10 protein. Secondary antibody staining was performed with 1:15000 LICOR goat anti-mouse (LI-COR). Imaging was done using a LI-COR Odyssey Imaging System (LI-COR).

\section{RNA-seq}

Animals were synchronized by hypochlorite treatment, propagated on NGM plates with OP50 at 16C, and harvested at the L4/young adult stage. Animals were washed off with N50, passed through a $5 \%$ sucrose cushion in N50 to remove E. coli, and snap frozen in liquid nitrogen. Animals were lysed by grinding in a mortar and pestle cooled in liquid nitrogen in the presence of frozen PLB (20mM Tris pH8.0, 140mM KCl, 1.5mM MgCl2, 1\% Triton) and 100ug/mL cycloheximide. Ground animals were stored as frozen powder at $-70 \mathrm{C}$.

Total RNA was extracted with trizol, resuspended in TE pH7.4, and quantified by Qubit.

890 Ribosomal RNA was depleted using custom C. elegans-specific rRNA hybridization oligos,

891 similar to a planarian protocol as described (Kim et al., 2019). Oligos for this protocol are

892 included in Table S1. Libraries were prepared using an NEBNext Ultra II Directional RNA

893 Library Prep Kit for Illumina sequencing. Libraries were sequenced at Novogene Corporation

894 Inc. UC Davis Sequencing Center on a NovaSeq 6000. 
Ribo-seq

897 For cycloheximide experiments, ground animals as described in RNA-seq were thoroughly

898 mixed with ice cold PLB and $100 \mathrm{ug} / \mathrm{mL}$ cycloheximide. For anisomycin experiments, animals

899 were ground in frozen PLB and $100 \mathrm{ug} / \mathrm{mL}$ anisomycin, then mixed with ice cold PLB and

$900100 \mathrm{ug} / \mathrm{mL}$ anisomycin. Ribosome::mRNA complexes were obtained via a 10 min clarifying spin

901 at 10,000 rcf at 4C and quantified via Nanodrop A260 measurement. Total OD units were

902 calculated and RNasel (Ambion) was added as per the following equation: total OD units $\times 0.3=$

903 ul RNasel. RNA was digested for $30 \mathrm{~min}$ at $23 \mathrm{C}$ and immediately loaded onto a chilled $10 \%-$

$90450 \%$ sucrose gradient containing $100 \mathrm{ug} / \mathrm{mL}$ cycloheximide or $100 \mathrm{ug} / \mathrm{mL}$ anisomycin.

Gradients were spun in an SW41 Ti rotor in an ultracentrifuge at 35,000 rpm for 4.5hrs at 4C.

Monosomes and disomes were collected on a fractionator (Brandel) and digested with proteinase K. Monosome and disome RNA was cleaned up by acid phenol chloroform extraction, precipitated, and stored in TE pH7.4. 2ug of purified monosome and disome RNA were run on $15 \%$ polyacrylamide gels and size-selected. For cycloheximide experiments, 2830nt (monosome) and 50-62nt (disome) footprints were excised. For anisomycin experiments, 18-30nt (monosome), 40-49nt (short disome), and 50-62nt (disome) footprints were excised. extracted with phenol chloroform, precipitated, and resuspended in TE pH7.4. Pre-adenylated adaptor containing 8nt UMI sequences (JA-AF-34.5) was ligated onto RNA 3'ends with T4 RNA Ligase 2 truncated KQ (NEB). Adaptor-ligated RNA was run on a 15\% polyacrylamide gel, and the ligated species was excised, eluted, and precipitated. Reverse transcription was performed using JA-AF-126 and superscript II RT (Thermo Fisher). cDNA was gel-purified and precipitated from a $15 \%$ polyacrylamide gel and circularized with CircLigase (Lucigen). Circularized DNA was amplified by PCR (10-14 cycles) using primers containing Illumina-compatible adaptors. Libraries were sequenced at Novogene Corporation Inc. UC Davis Sequencing Center on a NovaSeq 6000.

\section{RNA- and Ribo-seq analyses}

926 RNA- and Ribo-seq reads were trimmed using cutadapt v2.9 (Martin, 2011) and PCR duplicates 927 (identified by the 8nt UMI on AF-JA-34.5) were collapsed using custom scripts. We generated a 928 custom C. elegans genome (Ensembl, WBCel235) containing unc-54(rareArg) as a separate 929 chromosome and a masked endogenous unc-54 locus. Reads were mapped to this genome 930 including annotated splice junctions using STAR v2.5.4b (Dobin and Gingeras, 2015 26334920) 
931 allowing for zero mismatches. All downstream analyses were restricted to uniquely mapping

932 reads.

Analyses were performed in python3 and plotted using matplotlib or PyX v0.15 using custom

935 scripts. In rocket plots (e.g., Fig 3F), read counts were median-normalized using DESeq

936 (Anders and Huber, 2010).

Percent readthrough was calculated by finding the average footprints post-rareArg divided by

939 the average footprints pre-rareArg, normalized to the length. This was done omitting 100nts

940 upstream of the 5' end of a ribosome with the first arginine codon in its P-site and 100nts

941 downstream of the 5' end of a ribosome with the last arginine in its P-site. We note that this

942 metric will include effects such as 5 ' bias into the readthrough calculation; similar calculations

943 with smaller window sizes centered about the stall revealed similar results.

Meta-codon plots of footprint density around a specific motif were created similarly to that done previously (Meydan et al., 2020). Briefly, we generated a list of every occurrence of each amino acid triplet in the $C$. elegans transcriptome. Average normalized monosome and disome density

948 were then plotted by their 3' ends 50nt upstream and downstream of the first nucleotide of the

949 first amino acid in the triplet motif. Dotted lines were drawn at locations indicating the footprint 3'

950 end position when the third codon of each motif is in each ribosomal site.

\section{Ski7 search}

953 To complement a prior (Marshall et al., 2018) search for Ski7 homologs, we carried out a

954 thorough scan of the $C$. elegans genome to identify possible Ski7s. We performed a six-frame

955 translation of the genome and searched for matches to the S3 motif (FXX[P/A]SPD[D/E]) or the

$956 \mathrm{~N}$-term of HBS-1/Ski7 (M[A/S]RHR). These efforts failed to identify a protein with an S3 motif,

957 but did identify a single protein with an N-terminal M[A/S]RHR: h14e04.3. Further examination

958 of $h 14 e 04.3$ revealed a sequence coarsely resembling an S3 motif, but with loss of the central

959 "PD" motif. However, h14e04.3( $\Delta$ ) had no effect on Nonstop mRNA Decay (Fig S2). We note

960 that this approach may have failed to pick up a gene with introns in both the S3 and N-terminal

961 motif, but otherwise should have revealed a Ski7 with a canonical S3 motif, even if unannotated.

963 HBS-1 linker length calculation

964 Using the seed alignments from Pfam PF08938 (HBS-1 N-terminus domain) and PF00009

965 (elongation factor Tu GTP-binding domain), we constructed HMM profiles through hmmbuild. 
966 We used the resulting HMM profiles to search for HBS-1 homologs from genome database files.

967 A custom script identified proteins with hits from both domains, then calculated the distance in

968 between each domain (C-terminus of the $\mathrm{N}$-terminal domain to the $\mathrm{N}$-terminus of the GTP

969 binding domain). Initially, Uniref90 was used as a genome database, but due to the evolutionary

970 interrelatedness of $h b s-1$ and ski-7, many Ski7 proteins were misidentified as HBS-1. We were

971 able to identify suspected Ski7 proteins with the conserved S3 motif, a hallmark of Ski7 proteins

972 (Marshall et al., 2018). To overcome this issue, we ran the same script against a database of

973 Ensembl human HBS1L orthologs.

\section{Mutual information calculation}

976 Phylogenetic profiling was performed using the PANTHER HMM library Version 16.0 on

977 Ensembl Protists Genome (release 51) combined with MMETSP (Keeling et al., 2014). To

978 identify homologs of proteins from the PANTHER Subfamily HMMs, we used HMMER's

979 hmmsearch function using the following parameters --noali --notextw --cpu 2. We then parsed

980 each output file from HMMER and determined the presence (1) or absence (0) of a homolog in

981 each organism for a sub-family HMM.

983 Briefly, if multiple subfamily HMMs matched a given protein in a species, the protein was 984 assigned to the subfamily with the highest bit score, and lesser-scoring subfamilies were 985 ignored. Subfamilies with homologs in $<5 \%$ or $>95 \%$ of species were discarded; such

986 subfamilies exhibit too little variation for accurate mutual information calculations. Similarly, 987 species with hits for $<10 \%$ of subfamilies were discarded; such species may represent poor 988 protein assemblies. We also computed the pairwise hamming distance between all species and 989 discarded species until the pairwise hamming distance between all species was at least 0.1 , so 990 as to ensure biodiversity. Mutual information for discrete variables was then calculated as:

$991 \operatorname{sum}(i=0,1) ; \operatorname{sum}(j=0,1)\left[-\log \_2\left(p \_i j /\left(p \_i^{*} p \_j\right)\right)\right]$

994 Alam, S. L., Sun, J., Payne, M., Welch, B. D., Blake, B. K., Davis, D. R., Meyer, H. H., Emr, S. D., \& Sundquist, W. I. (2004). Ubiquitin interactions of NZF zinc fingers. EMBO Journal,

997 Anders, S., \& Huber, W. (2010). Differential expression analysis for sequence count data.

998 Genome biology, 11(10), R106. https://doi.org/10.1186/gb-2010-11-10-r106. PMID:

$999 \quad 20979621$ 
1000

1001

1002

1003

1004

1005

1006

1007

1008

1009

1010

1011

1012

1013

1014

1015

1016

1017

1018

1019

1020

1021

1022

1023

1024

1025

1026

1027

1028

1029

1030

1031

1032

1033

Araki, Y., Takahashi, S., Kobayashi, T., Kajiho, H., Hoshino, S. I., \& Katada, T. (2001). Ski7p G protein interacts with the exosome and the ski complex for $3^{\prime}$-to-5' mRNA decay in yeast. EMBO Journal, 20(17), 4684-4693. https://doi.org/10.1093/emboj/20.17.4684. PMID: 11532933

Arora, C., Kee, K., Maleki, S., \& Keeney, S. (2004). Antiviral protein Ski8 is a direct partner of Spo11 in meiotic DNA break formation, independent of its cytoplasmic role in RNA metabolism. Molecular Cell, 13(4), 549-559. https://doi.org/10.1016/s10972765(04)00063-2. PMID: 14992724

Arpat, A., Liechti, A., De Matos, M., Dreos, R., Janich, P., \& Gatfield, D. (2020). Transcriptomewide sites of collided ribosomes reveal principles of translational pausing. Genome Research, 30(7), 985-999. https://doi.org/10.1101/GR.257741.119. PMID: 32703885

Arribere, J. A., Bell, R. T., Fu, B. X., Artiles, K. L., Hartman, P. S., \& Fire, A. Z. (2014). Efficient marker-free recovery of custom genetic modifications with CRISPR/Cas 9 in Caenorhabditis elegans. Genetics, 198(3), 837-846. https://doi.org/10.1534/genetics.114.169730. PMID: 25161212

Arribere, J. A., \& Fire, A. Z. (2018). Nonsense mRNA suppression via nonstop decay. eLife, 7, 1-23. https://doi.org/10.7554/eLife.33292. PMID: 29309033

Becker, T., Armache, J. P., Jarasch, A., Anger, A. M., Villa, E., Sieber, H., Motaal, B. A., Mielke, T., Berninghausen, O., \& Beckmann, R. (2011). Structure of the no-go mRNA decay complex Dom34-Hbs1 bound to a stalled $80 \mathrm{~S}$ ribosome. Nature Structural and Molecular Biology, 18(6), 715-720. https://doi.org/10.1038/nsmb.2057. PMID: 21623367

Bengtson, M. H., \& Joazeiro, C. A. P. (2010). Role of a ribosome-associated E3 ubiquitin ligase in protein quality control. Nature, 467(7314), 470-473. https://doi.org/10.1038/nature09371. PMID: 20835226

Brandman, O., Stewart-Ornstein, J., Wong, D., Larson, A., Williams, C. C., Li, G. W., Zhou, S., King, D., Shen, P. S., Weibezahn, J., Dunn, J. G., Rouskin, S., Inada, T., Frost, A., \& Weissman, J. S. (2012). A ribosome-bound quality control complex triggers degradation of nascent peptides and signals translation stress. Cell, 151(5), 1042-1054. https://doi.org/10.1016/j.cell.2012.10.044. PMID: 23178123

Brenner S. (1974). The genetics of Caenorhabditis elegans. Genetics, 77(1), 71-94. https://doi.org/10.1093/genetics/77.1.71. PMID: 4366476

Cali, B. M., Kuchma, S. L., Latham, J., \& Anderson, P. (1999). smg-7 is required for mRNA surveillance in Caenorhabditis elegans. Genetics, 151(2), 605-616. https://doi.org/10.1093/genetics/151.2.605. PMID: 9927455 
1034 Carbone, C. E., Demo, G., Madireddy, R., Svidritskiy, E., \& Korostelev, A. A. (2020). ArfB can displace mRNA to rescue stalled ribosomes. Nature Communications, 11(1). https://doi.org/10.1038/S41467-020-19370-Z. PMID: 33144582

Chandrasekaran, V., Juszkiewicz, S., Choi, J., Puglisi, J. D., Brown, A., Shao, S., Ramakrishnan, V., \& Hegde, R. S. (2019). Mechanism of ribosome stalling during translation of a poly(A) tail. Nature Structural and Molecular Biology, 26(12), 1132-1140. https://doi.org/10.1038/s41594-019-0331-x. PMID: 31768042

Das, S., Vera, M., Gandin, V., Singer, R. H., \& Tutucci, E. (2021). Intracellular mRNA transport and localized translation. Nature Reviews Molecular Cell Biology, 22(7), 483-504. https://doi.org/10.1038/s41580-021-00356-8. PMID: 33837370

Dey, G., \& Meyer, T. (2015). Phylogenetic Profiling for Probing the Modular Architecture of the Human Genome. Cell Systems, 1(2), 106-115. https://doi.org/10.1016/J.CELS.2015.08.006. PMID: 27135799

Dmitriev, S. E., Vladimirov, D. O., \& Lashkevich, K. A. (2020). A Quick Guide to Small-Molecule Inhibitors of Eukaryotic Protein Synthesis. Biochemistry (Moscow), 85(11), 1389-1421. https://doi.org/10.1134/S0006297920110097. PMID: 33280581

Dobin, A., \& Gingeras, T. R. (2015). Mapping RNA-seq Reads with STAR. Current protocols in bioinformatics, 51, 11.14.1-11.14.19. https://doi.org/10.1002/0471250953.bi1114s51. PMID: 26334920

Doitsidou, M., Poole, R. J., Sarin, S., Bigelow, H., \& Hobert, O. (2010). C. elegans mutant identification with a one-step whole-genome-sequencing and SNP mapping strategy. PloS one, 5(11), e15435. https://doi.org/10.1371/journal.pone.0015435. PMID: 21079745

Doma, M. K., \& Parker, R. (2006). Endonucleolytic cleavage of eukaryotic mRNAs with stalls in translation elongation. Nature, 440(7083), 561-564. https://doi.org/10.1038/nature04530. PMID 16554824 
Edgar, R., Domrachev, M., \& Lash, A. E. (2002). Gene Expression Omnibus: NCBI gene expression and hybridization array data repository. Nucleic acids research, 30(1), 207210. https://doi.org/10.1093/nar/30.1.207. PMID: 11752295

Frischmeyer, P. A., Hoof, A. van, O’Donnell, K., Guerrerio, A. L., Parker, R., \& Dietz, H. C. (2002). An mRNA Surveillance Mechanism That Eliminates Transcripts Lacking Termination Codons. Science, 295(5563), 2258-2261. https://doi.org/10.1126/SCIENCE.1067338. PMID: 11910109

Garzia, A., Jafarnejad, S. M., Meyer, C., Chapat, C., Gogakos, T., Morozov, P., Amiri, M., Shapiro, M., Molina, H., Tuschl, T., \& Sonenberg, N. (2017). The E3 ubiquitin ligase and RNA-binding protein ZNF598 orchestrates ribosome quality control of premature polyadenylated mRNAs. Nature Communications, 8. https://doi.org/10.1038/ncomms16056. PMID: 28685749

Garzia, A., Meyer, C., \& Tuschl, T. (2021). The E3 ubiquitin ligase RNF10 modifies 40S ribosomal subunits of ribosomes compromised in translation. Cell Reports, 36(5), 109468. https://doi.org/10.1016/j.celrep.2021.109468. PMID: 34348161

Glover, M. L., Burroughs, A. M., Monem, P. C., Egelhofer, T. A., Pule, M. N., Aravind, L., \& Arribere, J. A. (2020). NONU-1 Encodes a Conserved Endonuclease Required for mRNA Translation Surveillance. Cell Reports, 30(13), 4321-4331.e4. https://doi.org/10.1016/j.celrep.2020.03.023. PMID: 32234470

Gutierrez, E., Shin, B. S., Woolstenhulme, C. J., Kim, J. R., Saini, P., Buskirk, A. R., \& Dever, T. E. (2013). elF5A promotes translation of polyproline motifs. Molecular Cell, 51(1), 35-45. https://doi.org/10.1016/j.molcel.2013.04.021. PMID: 23727016

Halbach, F., Reichelt, P., Rode, M., \& Conti, E. (2013). The yeast ski complex: Crystal structure and RNA channeling to the exosome complex. Cell, 154(4), 814-826. https://doi.org/10.1016/j.cell.2013.07.017. PMID: 23953113

Han, P., Shichino, Y., Schneider-Poetsch, T., Mito, M., Hashimoto, S., Udagawa, T., Kohno, K., Yoshida, M., Mishima, Y., Inada, T., \& Iwasaki, S. (2020). Genome-wide Survey of Ribosome Collision. Cell Reports, 31(5), 107610. https://doi.org/10.1016/j.celrep.2020.107610. PMID: 32375038 Hashimoto, Y., Takahashi, M., Sakota, E., \& Nakamura, Y. (2017). Nonstop-mRNA decay machinery is involved in the clearance of mRNA 5'-fragments produced by RNAi and NMD in Drosophila melanogaster cells. Biochemical and Biophysical Research Communications, 484(1), 1-7. https://doi.org/10.1016/j.bbrc.2017.01.092.

PMID:28115162 
1101 Hickey, K. L., Dickson, K., Cogan, J. Z., Replogle, J. M., Schoof, M., D’Orazio, K. N., Sinha, N. K., Hussmann, J. A., Jost, M., Frost, A., Green, R., Weissman, J. S., \& Kostova, K. K. (2020). GIGYF2 and 4EHP Inhibit Translation Initiation of Defective Messenger RNAs to Assist Ribosome-Associated Quality Control. Molecular Cell, 79(6), 950-962.e6. https://doi.org/10.1016/j.molcel.2020.07.007. PMID: 32726578

Hilal, T., Yamamoto, H., Loerke, J., Bürger, J., Mielke, T., \& Spahn, C. M. T. (2016). Structural insights into ribosomal rescue by Dom34 and Hbs1 at near-atomic resolution. Nature Communications, 7, 1-8. https://doi.org/10.1038/ncomms13521. PMID: 27995908

Hoof, A. van, Staples, R. R., Baker, R. E., \& Parker, R. (2000). Function of the Ski4p (Csl4p)

Hoof, A. van, Frischmeyer, P. A., Dietz, H. C., \& Parker, R. (2002). Exosome-Mediated Recognition and Degradation of mRNAs Lacking a Termination Codon. Science, 295(5563), 2262-2264. https://doi.org/10.1126/SCIENCE.1067272. PMID: 11910110

Ikeuchi, K., Tesina, P., Matsuo, Y., Sugiyama, T., Cheng, J., Saeki, Y., Tanaka, K., Becker, T., to induce Hel2-driven quality control pathways. The EMBO Journal, 38(5), 1-21. https://doi.org/10.15252/embj.2018100276. PMID: 30609991

Ishimura, R., Nagy, G., Dotu, I., Zhou, H., Yang, X. L., Schimmel, P., Senju, S., Nishimura, Y., Chuang, J. H., \& Ackerman, S. L. (2014). Ribosome stalling induced by mutation of a CNS-specific tRNA causes neurodegeneration. Science, 345(6195), 455-459. https://doi.org/10.1126/science.1249749. PMID: 25061210 Requires Site-Specific Ribosome Ubiquitination. Molecular Cell, 65(4), 743-750.e4. https://doi.org/10.1016/j.molcel.2016.11.039. PMID: 28065601

Juszkiewicz, S., Chandrasekaran, V., Lin, Z., Kraatz, S., Ramakrishnan, V., \& Hegde, R. S. (2018). ZNF598 Is a Quality Control Sensor of Collided Ribosomes. Molecular Cell, 72(3), 469-481.e7. https://doi.org/10.1016/j.molcel.2018.08.037. PMID: 30293783 ASC-1 Complex Disassembles Collided Ribosomes. Molecular Cell, 79(4), 603-614.e8. https://doi.org/10.1016/j.molcel.2020.06.006. PMID: 32579943

Kalisiak, K., Kuliński, T. M., Tomecki, R., Cysewski, D., Pietras, Z., Chlebowski, A., Kowalska, K., \& Dziembowski, A. (2017). A short splicing isoform of HBS1L links the cytoplasmic 
exosome and SKI complexes in humans. Nucleic Acids Research, 45(4), 2068-2080. https://doi.org/10.1093/nar/gkw862. PMID 28204585

Keeling, P. J., Burki, F., Wilcox, H. M., Allam, B., Allen, E. E., Amaral-Zettler, L. A., Armbrust, E. V., Archibald, J. M., Bharti, A. K., Bell, C. J., Beszteri, B., Bidle, K. D., Cameron, C. T., Campbell, L., Caron, D. A., Cattolico, R. A., Collier, J. L., Coyne, K., Davy, S. K., Deschamps, P., ... Worden, A. Z. (2014). The Marine Microbial Eukaryote

Kowalinski, E., Kögel, A., Ebert, J., Reichelt, P., Stegmann, E., Habermann, B., \& Conti, E. (2016). Structure of a Cytoplasmic 11-Subunit RNA Exosome Complex. Molecular Cell, 63(1), 125-134. https://doi.org/10.1016/j.molcel.2016.05.028. PMID: 27345150

Kulkarni, M., \& Smith, H. E. (2008). E1 ubiquitin-activating enzyme UBA-1 plays multiple roles throughout C. elegans development. PLoS Genetics, 4(7), 1000131. https://doi.org/10.1371/journal.pgen.1000131. PMID: 18636104

Lareau, L. F., Hite, D. H., Hogan, G. J., \& Brown, P. O. (2014). Distinct stages of the translation elongation cycle revealed by sequencing ribosome-protected mRNA fragments. eLife, 3, e01257. 1-16. https://doi.org/10.7554/eLife.01257. PMID: 24842990

Lu, J., \& Deutsch, C. (2008). Electrostatics in the Ribosomal Tunnel Modulate Chain Elongation Rates. Journal of Molecular Biology, 384(1), 73-86. https://doi.org/10.1016/j.jmb.2008.08.089. PMID: 18822297

Lu, J., Wu, T., Zhang, B., Liu, S., Song, W., Qiao, J., \& Ruan, H. (2021). Types of nuclear localization signals and mechanisms of protein import into the nucleus. Cell communication and signaling, 19(1), 60. https://doi.org/10.1186/s12964-021-00741-y. PMID: 34022911

Marshall, A. N., Han, J., Kim, M., \& Van Hoof, A. (2018). Conservation of mRNA quality control factor Ski7 and its diversification through changes in alternative splicing and gene duplication. Proceedings of the National Academy of Sciences of the United States of 
America, 115(29), E6808-E6816. https://doi.org/10.1073/pnas.1801997115. PMID: 29967155

Martin, M. (2011). Cutadapt removes adapter sequences from high-throughput sequencing reads. https://doi.org/10.14806/ej.17.1.200

Matsuo, Y., Tesina, P., Nakajima, S., Mizuno, M., Endo, A., Buschauer, R., Cheng, J., Shounai, O., Ikeuchi, K., Saeki, Y., Becker, T., Beckmann, R., \& Inada, T. (2020). RQT complex dissociates ribosomes collided on endogenous RQC substrate SDD1. Nature Structural and Molecular Biology, 27(4), 323-332. https://doi.org/10.1038/s41594-020-0393-9. PMID: 32203490

Meydan, S., \& Guydosh, N. R. (2020). Disome and Trisome Profiling Reveal Genome-wide Targets of Ribosome Quality Control. Molecular Cell, 79(4), 588-602.e6. https://doi.org/10.1016/j.molcel.2020.06.010. PMID: 32615089

McKenna, A., Hanna, M., Banks, E., Sivachenko, A., Cibulskis, K., Kernytsky, A., Garimella, K., Altshuler, D., Gabriel, S., Daly, M., \& DePristo, M. A. (2010). The Genome Analysis Toolkit: a MapReduce framework for analyzing next-generation DNA sequencing data. Genome research, 20(9), 1297-1303. https://doi.org/10.1101/gr.107524.110. PMID: 20644199

Mi, H., Ebert, D., Muruganujan, A., Mills, C., Albou, L. P., Mushayamaha, T., \& Thomas, P. D. (2021). PANTHER version 16: a revised family classification, tree-based classification tool, enhancer regions and extensive API. Nucleic Acids Research, 49(D1), D394-D403. https://doi.org/10.1093/nar/gkaa1106. PMID: 33290554

Mueller, T. D., \& Feigon, J. (2002). Solution Structures of UBA Domains Reveal a Conserved Hydrophobic Surface for Protein-Protein Interactions. Journal of Molecular Biology, 319(5), 1243-1255. https://doi.org/10.1016/S0022-2836(02)00302-9. PMID: 12079361

Navickas, A., Chamois, S., Saint-Fort, R., Henri, J., Torchet, C., \& Benard, L. (2020). No-Go Decay mRNA cleavage in the ribosome exit tunnel produces 5 '-OH ends phosphorylated by Trl1. Nature Communications, 11(1), 1-11. https://doi.org/10.1038/s41467-01913991-9. PMID: 31913314

Pisareva, V. P., Skabkin, M. A., Hellen, C. U. T., Pestova, T. V., \& Pisarev, A. V. (2011). Dissociation by Pelota, Hbs1 and ABCE1 of mammalian vacant $80 \mathrm{~S}$ ribosomes and stalled elongation complexes. EMBO Journal, 30(9), 1804-1817. https://doi.org/10.1038/emboj.2011.93. PMID: 21448132

Prag, G., Misra, S., Jones, E. A., Ghirlando, R., Davies, B. A., Horazdovsky, B. F., \& Hurley, J. H. (2003). Mechanism of ubiquitin recognition by the CUE domain of Vps9p. Cell, 113(5), 609-620. https://doi.org/10.1016/s0092-8674(03)00364-7. PMID: 12787502 
1205

1206

1207

1208

1209

1210

1211

1212

1213

1214

1215

1216

1217

1218

1219

1220

1221

1222

1223

1224

1225

1226

1227

1228

1229

1230

1231

1232

1233

1234

1235

1236

1237

1238

Randles, L., \& Walters, K. J. (2012). Ubiquitin and its binding domains. Frontiers in Bioscience, 17, 2140-2157. https://doi.org/10.2741/4042. PMID: 22652769

Roach, N. P., Sadowski, N., Alessi, A. F., Timp, W., Taylor, J., \& Kim, J. K. (2020). The fulllength transcriptome of $C$. elegans using direct RNA sequencing. Genome research, 30(2), 299-312. https://doi.org/10.1101/gr.251314.119 PMID: 32024661

Ryabova, L. A., \& Hohn, T. (2000). Ribosome shunting in the cauliflower mosaic virus 35S RNA leader is a special case of reinitiation of translation functioning in plant and animal systems. Genes \& development, 14(7), 817-829. PMID: 10766738

Saito, S., Hosoda, N., \& Hoshino, S. I. (2013). The Hbs1-Dom34 protein complex functions in non-stop mRNA decay in mammalian cells. Journal of Biological Chemistry, 288(24), 17832-17843. https://doi.org/10.1074/jbc.M112.448977. PMID: 23667253

Schmidt, C., Becker, T., Heuer, A., Braunger, K., Shanmuganathan, V., Pech, M., Berninghausen, O., Wilson, D. N., \& Beckmann, R. (2016). Structure of the hypusinylated eukaryotic translation factor elF-5A bound to the ribosome. Nucleic Acids Research, 44(4), 1944-1951. https://doi.org/10.1093/nar/gkv1517. PMID: 26715760

Schuller, A. P., Wu, C. C., Dever, T. E., Buskirk, A. R., \& Green, R. (2017). elF5A Functions Globally in Translation Elongation and Termination. Molecular Cell, 66(2), 194-205.e5. https://doi.org/10.1016/j.molcel.2017.03.003. PMID: 28392174

Shao, S., \& Hegde, R. S. (2014). Reconstitution of a Minimal Ribosome-Associated Ubiquitination Pathway with Purified Factors. Molecular Cell, 55(6), 880-890. https://doi.org/10.1016/j.molcel.2014.07.006. PMID: 25132172

Shoemaker, C. J., Eyler, D. E., \& Green, R. (2010). Dom34:Hbs1 Promotes Subunit Dissociation and Peptidyl-tRNA Drop-Off to Initiate No-Go Decay. Science, 330(6002), 369-372. https://doi.org/10.1126/SCIENCE.1192430. PMID: 20947765

Shoemaker, C. J., \& Green, R. (2011). Kinetic analysis reveals the ordered coupling of translation termination and ribosome recycling in yeast. Proceedings of the National Academy of Sciences of the United States of America, 108(51). https://doi.org/10.1073/pnas.1113956108. PMID: 22143755

Simms, C. L., Yan, L. L., \& Zaher, H. S. (2017). Ribosome Collision Is Critical for Quality Control during No-Go Decay. Molecular Cell, 68(2), 361-373.e5. https://doi.org/10.1016/j.molcel.2017.08.019. PMID: 28943311

Simms, C. L., Yan, L. L., Quu, J. K., \& Zaher, H. S. (2019). Ribosome Collisions Result in +1 Frameshifting in the Absence of No-Go Decay. Cell Reports, 28(7), 1679-1689.e4. https://doi.org/10.1016/j.celrep.2019.07.046. PMID: 31412239 
Stadler, M., \& Fire, A. Z. (2011). Wobble base-pairing slows in vivo translation elongation in metazoans. RNA, 17(12), 2063-2073. https://doi.org/10.1261/RNA.02890211. PMID: 22045228

Sundaramoorthy, E., Leonard, M., Mak, R., Liao, J., Fulzele, A., \& Bennett, E. J. (2017). ZNF598 and RACK1 Regulate Mammalian Ribosome-Associated Quality Control Function by Mediating Regulatory 40S Ribosomal Ubiquitylation. Molecular Cell, 65(4), 751-760.e4. https://doi.org/10.1016/j.molcel.2016.12.026. PMID: 28132843

Thompson, O., Edgley, M., Strasbourger, P., Flibotte, S., Ewing, B., Adair, R., Au, V., Chaudhry, I., Fernando, L., Hutter, H., Kieffer, A., Lau, J., Lee, N., Miller, A., Raymant, G., Shen, B., Shendure, J., Taylor, J., Turner, E. H., Hillier, L. W., ... Waterston, R. H. (2013). The million mutation project: a new approach to genetics in Caenorhabditis elegans. Genome research, 23(10), 1749-1762. https://doi.org/10.1101/gr.157651.113. PMID: 23800452

Wang, J., Zhou, J., Yang, Q., \& Grayhack, E. J. (2018). Multi-protein bridging factor 1(Mbf1), Rps3 and Asc1 prevent stalled ribosomes from frameshifting. eLife, 7, 1-26. https://doi.org/10.7554/eLife.39637. PMID: 30465652

Zhao, T., Chen, Y.-M., Li, Y., Wang, J., Chen, S., Gao, N., \& Qian, W. (2021). Disome-seq reveals widespread ribosome collisions that promote cotranslational protein folding. Genome Biology, 22(1), 1-35. https://doi.org/10.1186/S13059-020-02256-0. PMID: 33402206 